\title{
The mathematical theory of thermal choking in nozzle flows
}

By Can F. Delale†, Institut für Strömungslehre und Strömungsmaschinen, Universität (TH) Karlsruhe and Dept of Mathematics, Bilkent University, Ankara, Turkey and Günter H. Schnerr and Jürgen Zierep, Institut für Strömungslehre und Strömungsmaschinen, Universität (TH) Karlsruhe, Germany

\section{Introduction}

Heat addition to flows expanding through a Laval nozzle can be achieved in a number of ways such as by exothermic chemical reactions, phase change, etc. The classical gas dynamic equations for nozzle flows then need to be supplemented by the corresponding nonequilibrium rate equations which give rise to internal heat release. Usually such a system of equations appears complicated to analyze, and one instead chooses to treat the amount of internal heat release as a given function of the nozzle axial coordinate. Such flows will be termed diabatic. A comprehensive discussion of diabatic flows is already available in the literature from the work of Shapiro (1953), Jungclaus and van Raay (1967), Möhring (1979), Zierep (1990) and its application to nozzle flows with nonequilibrium condensation can be found in Wegener and Mack (1958), Pouring (1965) and Barschdorff (1967). It is well-known that when the amount of internal heat release exceeds a certain limit the phenomenon usually referred to as thermal choking occurs. The flow in this case is visualized by the existence of normal shock waves and is termed supercritical. It is commonly believed that the flow Mach number reaches unity at thermal choking and the critical amount of heat not to be exceeded in shock free flows is usually obtained by application of the corresponding expression for diabatic flows in channels of constant area [Wegener and Mack (1958)]. A satisfactory theory of thermal choking in nozzle flows seems lacking.

It is the purpose of this study to lay down the fundamentals of such a theory for diabatic nozzle flows and for flows with nonequilibrium conden-

$\uparrow$ Alexander von Humboldt Fellow. 
sation. For this reason the expression for the critical amount of heat necessary for thermal choking is defined and models that approximate this expression are constructed for both diabatic nozzle flows and flows with nonequilibrium condensation. In particular for condensing nozzle flows a cubic equation for an estimate of the limiting condensate mass fraction in shock free flows is derived and agreement with experimentally visualized results is achieved. Consequently a decisive criterion for sub- and supercritical flows is established. The necessary and sufficient conditions for thermal choking are then stated. It is shown that the classical view which assumes that the flow Mach number be unity at thermal choking may not hold in general for condensing flows in the widely adopted two-phase homogeneous dispersed flow model unless the latent heat of condensation is treated as a constant.

\section{Integro-algebraic theory of nozzle flows with heat addition from phase change and internal sources}

We consider the transonic flow of a mixture of a carrier gas (herein denoted by subscript $i$ ) and a condensible vapor (herein denoted by subscript $v$ ) through a converging-diverging Laval nozzle with initial reservoir temperature $T_{0}^{\prime}$, initial specific humidity $\omega_{0}$, initial relative humidity $\phi_{0}$ and with geometry as shown in Fig. 1. We let $x^{\prime}$ denote the axial coordinate with origin at the throat and assume that the state of the vapor crosses the co-existence line at a location $x_{s}^{\prime}$ called the saturation point. We nondimensionalize the axial coordinate by $x \equiv x^{\prime} / 2 y^{*}$ where $2 y^{*}$ is the height of the throat. We further normalize the mixture pressure $p^{\prime}$, the mixture temperature $T^{\prime}$ and the mixture density $\varrho^{\prime}$ by choosing the saturation state, herein denoted by subscript $s$, as a reference state:

$$
p \equiv \frac{p^{\prime}}{p_{s}^{\prime}}, \quad T \equiv \frac{T^{\prime}}{T_{s}^{\prime}}, \quad \varrho \equiv \frac{\varrho^{\prime}}{\varrho_{s}^{\prime}}
$$

where $\varrho_{s}^{\prime}, T_{s}^{\prime}$ and $p_{s}^{\prime}$ are respectively the mixture density, the mixture temperature and the mixture pressure at the saturation point. The area is

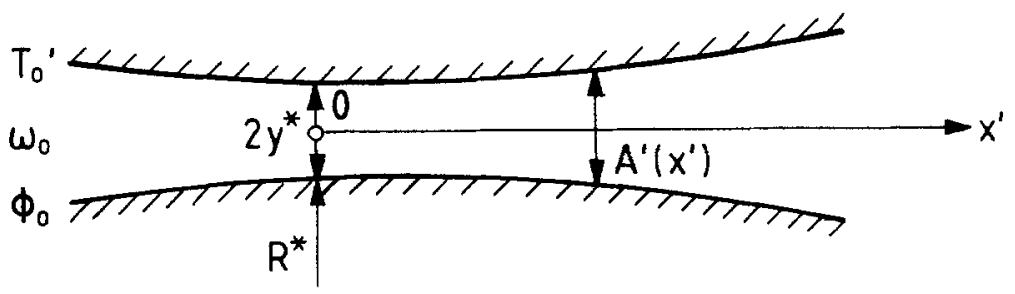

Figure 1

Geometry of a Laval nozzle. 
normalized in a similar manner as

$$
A(x) \equiv \frac{A^{\prime}(x)}{A^{\prime}\left(x_{s}\right)}
$$

where $x$ is the normalized axial coordinate and $x_{s}$ is its value at saturation. The normalization of the flow velocity $u^{\prime}$ is carried out somewhat differently by

$$
u \equiv \frac{u^{\prime}}{\left(\Re T_{s}^{\prime} / \mu_{0}\right)^{1 / 2}}
$$

where $\mathfrak{R}$ is the universal gas constant and $\mu_{0}$ is the molecular mass of the mixture defined by

$$
\mu_{0}^{-1} \equiv \omega_{0} \mu_{v}^{-1}+\left(1-\omega_{0}\right) \mu_{i}^{-1}
$$

with $\mu_{i}, \mu_{v}$ respectively denoting the molecular masses of the carrier gas and of the condensible vapor. We also let $g^{\prime}$, defined by

$$
g^{\prime} \equiv \frac{m_{c o n}^{\prime}}{m_{m i x}^{\prime}}
$$

where $m_{m i x}^{\prime}$ and $m_{c o n}^{\prime}$ are respectively the mass flow rates of the mixture and of the condensed phase, denote the nonequilibrium condensate mass fraction, $L^{\prime}(T)$ denote the latent heat of condensation at some normalized temperature $T$ and $Q_{\text {in }}^{\prime}(x)$ denote the total amount of heat added to the flow from internal sources at any location $x$. We further define $c_{p 0}$, the normalized specific heat at constant pressure, by

$$
c_{p 0} \equiv \frac{\gamma}{\gamma-1} \equiv \frac{\mu_{0} c_{p 0}^{\prime}}{\mathfrak{R}}
$$

where $\gamma$ is the isentropic exponent and $c_{p 0}^{\prime}$ is the mixture specific heat at constant pressure given by

$$
c_{p 0}^{\prime} \equiv \omega_{0} c_{p v}^{\prime}+\left(1-\omega_{0}\right) c_{p i}^{\prime}
$$

with $c_{p v}^{\prime}$ and $c_{p i}^{\prime}$ denoting respectively the specific heats at constant pressure of the vapor and of the carrier gas. With the above normalization and the conventional assumptions used for modelling the two-phase dispersed flow mixture (e.g. see Wegener [1969]), the mixture flow and state equations for steady one-dimensional nozzle flows with heat addition from nonequilibrium condensation and internal sources can be conveniently written as

$$
\begin{aligned}
& \varrho u A=u_{s} \\
& p A+\varrho u^{2} A=1+u_{s}^{2}+R\left(g^{\prime}, x\right) \\
& c_{p 0} T+\frac{1}{2} u^{2}-q\left(g^{\prime}, x\right)=c_{p 0} T_{0} \\
& p=\varrho T\left(1-\frac{\mu_{0}}{\mu_{v}} g^{\prime}\right)
\end{aligned}
$$


where the conventional momentum differential equation is replaced by the integral momentum theory, Eq. (2), and where $q\left(g^{\prime}, x\right)$ and $R\left(g^{\prime}, x\right)$ are defined by

$$
\begin{aligned}
& q\left(g^{\prime}, x\right) \equiv \frac{g^{\prime} L^{\prime}(T)+Q_{\text {in }}^{\prime}(x)}{\mathfrak{R} T_{s}^{\prime} / \mu_{0}} \\
& R\left(g^{\prime}, x\right) \equiv \int_{x_{s}}^{x} p\left(g^{\prime}(\xi), \xi\right) \frac{d A}{d \xi} d \xi
\end{aligned}
$$

and $T_{0}=T_{0}^{\prime} / T_{s}^{\prime}$. It is obvious that the system of equations given by (1)-(6) does not form a complete system since no consideration of the nonequilibrium condensation rate equation is yet given. The nonequilibrium condensation rate equation is very complicated and requires certain assumptions about the nature of condensation nuclei formation and their subsequent growth together with a knowledge of some thermodynamic properties of both the condensible vapor and the condensed phase which are generally poorly known. In spite of this fact Hill (1966) has carried out a detailed analysis of the physical mechanisms underlying the nature of the rate processes required for the construction of the nonequilibrium condensation rate equation. Asymptotic theories of the nonequilibrium condensation rate equation for nozzle flows (with different ordering of the double-limit process corresponding to large nucleation time followed by small droplet growth time) that yield the structure of condensation zones are already available from the work of Blythe and Shih (1976) and Clarke and Delale (1986). Results of experiments conducted in nozzle flows during the expansion of moist air and pure steam which reveal some general features about the physics of nonequilibrium condensation can also be found in the work of Wegener and Mack (1958), Pouring (1965), Barschdorff (1967), Wegener (1969), Schnerr (1989) and in their extensive references.

However, in what follows no discussion of the condensation rate equation, except for some well-known general features that have already been verified both theoretically and experimentally, is needed for the analysis of thermal choking and thereby we can proceed without loss of generality by assuming that $g^{\prime}=g^{\prime}(x)$ is an arbitrary, positive, strictly increasing function for $x>x_{s}$.

The system of equations (1)-(4) can then be solved for the flow velocity $u$ in functional form as

$$
u\left(g^{\prime}, x\right)=\frac{\left\{\left[1+u_{s}^{2}+R\left(g^{\prime}, x\right)\right] /\left(2 u_{s}\right)\right\} \pm \sqrt{\Delta\left(g^{\prime}, x\right)}}{\left[\gamma+1+(\gamma-1) \frac{\mu_{0}}{\mu_{v}} g^{\prime}\right] /(2 \gamma)}
$$


where $\Delta\left(g^{\prime}, x\right)$ is defined by

$$
\begin{aligned}
\Delta\left(g^{\prime}, x\right) \equiv & \left(\frac{1+u_{s}^{2}+R\left(g^{\prime}, x\right)}{2 u_{s}}\right)^{2} \\
& -\left(\frac{\gamma+1+(\gamma-1) \frac{\mu_{0}}{\mu_{v}} g^{\prime}}{2 \gamma}\right)\left(1-\frac{\mu_{0}}{\mu_{v}} g^{\prime}\right)\left(T_{0}+\frac{q\left(g^{\prime}, x\right)}{c_{p 0}}\right) .
\end{aligned}
$$

The remaining flow variables $\varrho, p$ and $T$ can then be given in functional form in terms of $u\left(g^{\prime}, x\right)$ as

$$
\begin{aligned}
& \varrho\left(g^{\prime}, x\right)=\frac{u_{s}}{u\left(g^{\prime}, x\right) A(x)} \\
& p\left(g^{\prime}, x\right)=\frac{1+u_{s}^{2}+R\left(g^{\prime}, x\right)-u_{s} u\left(g^{\prime}, x\right)}{A(x)} \\
& T\left(g^{\prime}, x\right)=T_{0}+\frac{q\left(g^{\prime}, x\right)}{c_{p 0}}-\frac{\left[u\left(g^{\prime}, x\right)\right]^{2}}{2 c_{p 0}} .
\end{aligned}
$$

In Eqs. (7) $-(11) Q_{\text {in }}^{\prime}(x)$ and $A(x)$ are assumed given. However, neither the function $g^{\prime}(x)$ nor the impulse function $R\left(g^{\prime}, x\right)$ nor the latent heat of condensation $L^{\prime}(T)$ are known. Even if $g^{\prime}(x)$ were exactly supplied by experiment or any other means, Equations (7)-(11) would still not constitute a local solution of Eqs. (1)-(4) since $L^{\prime}(T)$ and $R\left(g^{\prime}, x\right)$ remain to be evaluated. Indeed for any given strictly increasing function $g^{\prime}(x)$, Eqs. (7)-(11)--just like Eqs. (1)-(4) --form a system of integro-algebraic equations for the flow variables $\varrho, p, T$ and $u$. This means that Eqs. (7) - (11) do not completely decouple the rate equation from the flow and state equations (1)-(4). Thus it seems nothing special has yet been gained from the integro-algebraic formulation of nozzle flows. However, when for some given $g^{\prime}(x)$ certain approximations for $R\left(g^{\prime}, x\right)$ and $L^{\prime}(T)$ are made, some flow models which possess an approximate solution of Eqs. (1)-(4) given by Eqs. (7) $-(11)$ can be constructed. Furthermore the actual solution of the system of Eqs. (1)-(4) can in principle be obtained from these approximate solutions by iteration. Thus we proceed by applying the integro-algebraic formulation to different nozzle flow regimes to obtain either exact or approximate solutions of Eqs. (1)-(4).

\subsection{Isentropic nozzle flows}

For isentropic nozzle flows we set $g^{\prime} \equiv 0$ and $Q_{\text {in }}^{\prime}(x) \equiv 0$. In this case the system of Eqs. (1)-(4) form a complete system. We herein show how Eqs. (7) $-(11)$ for isentropic nozzle flows reduce to the exact classical solution. Before we proceed any further, we first note that the mixture of gases should 
now be interpreted as a mixture of two noncondensible gases. In this case the saturation reference state has no physical basis and we assume that normalization is carried out with respect to any other reference state of our choice to be denoted by subscript $b$. Thus in the preceding formulation we replace subscript $s$ by subscript $b$. In this case the impulse function $R$ and the function $\Delta$ in Eqs. (7) -(11) can be exactly evaluated from the isentropic nozzle flow relations as

$$
\begin{aligned}
& R \equiv R_{i s}(x)=\frac{M_{b}}{M(x)} \sqrt{\frac{1+\frac{(\gamma-1)}{2} M_{b}^{2}}{1+\frac{(\gamma-1)}{2} M^{2}(x)}}\left(1+\gamma M^{2}(x)\right)-\gamma M_{b}^{2}-1 \\
& \Delta \equiv \Delta_{i s}(x)=\frac{\left[1+\frac{(\gamma-1)}{2} M_{b}^{2}\right]\left(M^{2}(x)-1\right)^{2}}{4 \gamma M^{2}(x)\left[1+\frac{(\gamma-1)}{2} M^{2}(x)\right]}
\end{aligned}
$$

where $M(x)$ is the local isentropic flow Mach number, $M_{b}$ is its value at the chosen reference state $b, u_{b}^{2}=\gamma M_{b}^{2}$ and where the subscript $i s$ denotes the isentropic flow solution. We first note that $\Delta_{i s}(x) \geq 0$ for all $x$. In particular $\Delta_{i s}(x)=0$ if and only if $M(x)=1$, i.e. at the throat $(x=0)$. It follows directly from Eq. (7) for isentropic flows that

$$
u_{i s}(x)=M(x) \sqrt{\gamma \frac{1+\frac{(\gamma-1)}{2} M_{b}^{2}}{1+\frac{(\gamma-1)}{2} M^{2}(x)}}
$$

provided that in Eq. (7) the + sign is chosen for the square root whenever $M(x)>1$ and the - sign is chosen whenever $M(x)<1$. Equation (14) is precisely the classical isentropic solution for $u$ (which defines the local speed of sound) where normalization is carried out with respect to the reference state $b$. In particular in the limit $M \rightarrow 1$, we recover the expression for the normalised local speed of sound at the throat as

$$
u_{i s}(0)=\sqrt{\frac{2 \gamma}{\gamma+1}\left[1+\frac{(\gamma-1)}{2} M_{b}^{2}\right]} .
$$

By substituting from Eq. (14) into Eqs. (9) -(11) we arrive at the classical isentropic flow solution in the above normalization for the remaining flow variables as well. Thus we have verified that Eqs. (7)-(11) together with Eqs. (12) $-(13)$ indeed satisfy the classical isentropic nozzle flow relations [e.g. see Thompson (1972)]. 


\subsection{Diabatic nozzle flows}

We now investigate nozzle flows with internal heat addition from known distributed sources. We set $g^{\prime} \equiv 0$ and $Q_{\text {in }}^{\prime}(x) \equiv 0$ for $x \leq x_{b}$ and $Q_{\text {in }}^{\prime}(x)>0$ for $x>x_{b}$ where $Q_{\text {in }}^{\prime}(x)$ is continuous and strictly increasing for $x>x_{b}$ and where $x_{b}$ denotes the point where heat addition from internal sources has started. We further choose the state at $x=x_{b}$ as our reference state for normalization and thus replace subscript $s$ by subscript $b$ as in Section 2.1. We then obtain at once from Eq. (8) that

$$
\Delta \equiv \Delta_{d i}(x)=\left(\frac{1+u_{b}^{2}+R_{d i}(x)}{2 u_{b}}\right)^{2}-\frac{(\gamma+1)}{2 \gamma} T_{0}\left(1+\frac{Q(x)}{c_{p 0} T_{0}}\right)
$$

where

$$
\frac{Q(x)}{c_{p 0} T_{0}} \equiv \frac{Q_{\text {in }}^{\prime}(x)}{c_{p 0}^{\prime} T_{0}^{\prime}}
$$

and

$$
R_{d i}(x) \equiv \int_{x_{b}}^{x} p_{d i}(\xi) \frac{d A}{d \xi} d \xi
$$

with the subscript $d i$ referring to diabatic flow conditions. In particular $\Delta_{d i}(x)=\Delta_{i s}(x)$ and $R_{d i}(x)=R_{i s}(x)$ for $x \leq x_{b}$. For $x>x_{b}, R_{d i}(x)$ remains unknown and should be evaluated from Eq. (18) in order that Eqs. (7) -(11) represent the diabatic nozzle flow solution. It follows at once from Eq. (7) that for a continuous solution we must fulfill the condition $\Delta_{d i}(x) \geq 0$ for all $x>x_{b}$ which is equivalent to

$$
\frac{Q(x)}{c_{p 0} T_{0}} \leq \frac{Q^{*}(x)}{c_{p 0} T_{0}}
$$

for all $x>x_{b}$ where $Q^{*}(x)$, the critical amount of heat not to be exceeded at any $x$, is defined by

$$
\frac{Q^{*}(x)}{c_{p 0} T_{0}} \equiv \frac{2 \gamma}{(\gamma+1) T_{0}}\left(\frac{1+u_{b}^{2}+R_{d i}(x)}{2 u_{b}}\right)^{2}-1 .
$$

We now define subcritical and supercritical diabatic flows. We call a diabatic nozzle flow subcritical if

$$
\frac{Q(x)}{c_{p 0} T_{0}}<\frac{Q^{*}(x)}{c_{p 0} T_{0}}
$$

for all $x>x_{b}$ and supercritical if

$$
\frac{Q(x)}{c_{p 0} T_{0}}>\frac{Q^{*}(x)}{c_{p 0} T_{0}}
$$

for some $x>x_{b}$. It follows immediately from this definition and inequality (19) that supercritical diabatic flows do not admit a continuous solution and 
are always realized with normal shock waves. An interesting question that can be posed now is whether or not for given initial reservoir conditions, nozzle geometry and internal heat addition the flow will be supercritical. The answer to this question lies whether or not under given conditions inequality (19) is violated at any $x$. This requires evaluation of $Q^{*}(x)$ given by Eq. (20). For $x \leq x_{b}$ the evaluation of $Q^{*}(x)$ is simple since $R_{d i}(x)=R_{i s}(x)$, but not useful. The result at any location $x \leq x_{b}$ is the same as the classical diabatic flow result at fixed constant area $A(x)$ continued downstream:

$$
\frac{Q^{*}(x)}{c_{p 0} T_{0}}=\frac{\left(M^{2}(x)-1\right)^{2}}{2(\gamma+1) M^{2}(x)\left(1+\frac{(\gamma-1)}{2} M^{2}(x)\right)}>0 .
$$

The exact evaluation of $Q^{*}(x) /\left(c_{p 0} T_{0}\right)$ for $x>x_{b}$ requires knowledge of $R_{d i}(x)$ which is presently unknown. In order to proceed further we discuss some diabatic nozzle flow models by approximating $R_{d i}(x)$ for $x>x_{b}$.

Model I [Wegener and Mack (1958)]. In this model we assume $R_{d i}(x)=R_{d i}\left(x_{b}\right)=0$ for $x>x_{b}$. This model can be physically realized by truncating the nozzle at $x=x_{b}$ and continuing downstream with heat addition from internal sources at constant area $A_{b} \equiv A\left(x_{b}\right)$ (Fig. 2). It follows directly from Eq. (20) that the critical amount of heat in this model, herein denoted by $Q_{I}^{*}(x)$, for $x>x_{b}$ is a constant given by

$$
\frac{Q_{I}^{*}(x)}{c_{p 0} T_{0}}=\frac{\left(M_{b}^{2}-1\right)^{2}}{2(\gamma+1) M_{b}^{2}\left(1+\frac{(\gamma-1)}{2} M_{b}^{2}\right)}<\frac{Q^{*}(x)}{c_{p o} T_{0}} .
$$

We further note that for $x>x_{b}$ the function $\Delta$, denoted by $\Delta_{I}(x)$ in this model, can be evaluated from Eq. (16) as

$$
\begin{aligned}
\Delta_{I}(x) & =\frac{\left(M_{b}^{2}-1\right)^{2}-2(\gamma+1) M_{b}^{2}\left(1+\frac{(\gamma-1)}{2} M_{b}^{2}\right)\left(Q(x) /\left(c_{p 0} T_{0}\right)\right)}{4 \gamma M_{b}^{2}} \\
& <\Delta_{d i}(x) .
\end{aligned}
$$

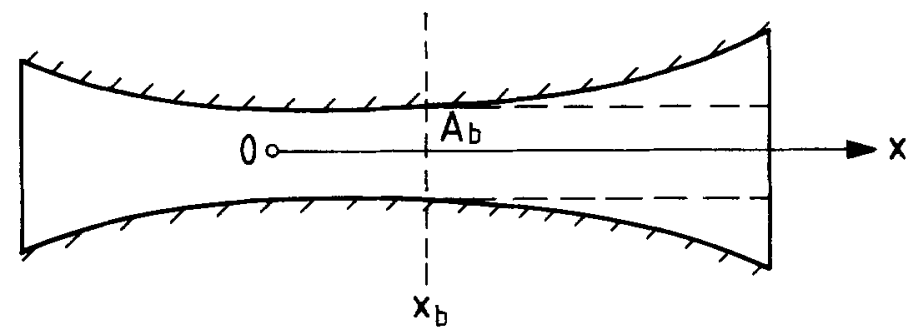


In this model all the flow variables for $x>x_{b}$ can now be evaluated from Eqs. (7) $-(11)$ by taking $g^{\prime} \equiv 0, R=0, q /\left(c_{p 0} T_{0}\right)=Q(x) /\left(c_{p 0} T_{0}\right), \Delta=\Delta_{I}(x)$ and by replacing subscript $s$ by subscript $b$.

Model II. This model is defined by setting $R_{d i}(x)=R_{i s}(x)$ for all $x$. It seems that this model can not be physically realized as a diabatic model since heat addition without increase of entropy is impossible; however, when the entropy increase due to internal heat addition within the accuracy of the governing equations remains smaller in order of magnitude than the changes in the rest of the flow variables, the model characterizes transonic small disturbance theory [e.g. see Zierep (1965)]. In this model the critical amount of heat, denoted by $Q_{I I}^{*}(x)<Q^{*}(x)$, is given by Eq. (21) for all $x$. The function $\Delta$, denoted by $\Delta_{I I}(x)$ in this model, evaluates to

$$
\begin{aligned}
\Delta_{I I}(x)= & \frac{1+\frac{(\gamma-1)}{2} M_{b}^{2}}{4 \gamma M^{2}(x)\left(1+\frac{(\gamma-1)}{2} M^{2}(x)\right)} \\
& \times\left(\left[M^{2}(x)-1\right]^{2}-2(\gamma+1) M^{2}(x)\right. \\
& \left.\times\left[1+\frac{(\gamma-1)}{2} M^{2}(x)\right] Q(x) /\left(c_{p 0} T_{0}\right)\right) \\
\leq & \Delta_{d i}(x)
\end{aligned}
$$

for all $x$. The flow variables at any location $x$ in this model follow from Eqs. (7) - (11) by taking $g^{\prime} \equiv 0, \Delta=\Delta_{I I}(x), R=R_{i s}(x), q /\left(c_{p 0} T_{0}\right)=Q(x) /\left(c_{p 0} T_{0}\right)$ and by replacing subscript $s$ by subscript $b$.

By comparing the above models with the actual diabatic flow, we immediately arrive at the following results which are fairly easy to prove:

Proposition 2.2.1. $Q^{*}(x)$ and $Q_{I I}^{*}(x)$ are strictly decreasing for $x<0$ $(d A / d x<0)$ and strictly increasing for $x>0(d A / d x>0)$. $Q_{I}^{*}(x)$ is strictly decreasing for $x<x_{b}$ and is a constant for $x>x_{b}$ whenever $x_{b} \leq 0 ; Q_{i}^{*}(x)$ is strictly decreasing for $x<0$, strictly increasing for $0<x<x_{b}$ and is a constant for $x>x_{b}$ whenever $x_{b}>0$.

Proposition 2.2.2. For $x>x_{b}>0$

$$
Q^{*}(x)>Q_{I I}^{*}(x)>Q_{I}^{*}(x)
$$

and

$$
\Delta_{d i}(x)>\Delta_{I I}(x)>\Delta_{I}(x) .
$$


From the above propositions it seems that Model II is a better approximation to the actual diabatic flow. In principle the actual diabatic flow can be computed from Model II by iteration. The algorithm is fairly simple: Given initial reservoir conditions, nozzle geometry and $Q_{\text {in }}^{\prime}(x)$, one evaluates the flow variables from Model II for all $x$. Using the pressure distribution from this model, one obtains the first approximation to the functions $R_{d i}(x)$ and $\Delta_{d i}(x)$ from Eqs. (18) and (16) respectively. Substituting the first approximation to the functions $R_{d i}(x)$ and $\Delta_{d i}(x)$ in Eqs. (7)-(11), one arrives at the first approximation for diabatic flow variables. Continuing from the pressure distribution of the first approximation in the same manner, one arrives at higher approximations for the diabatic flow variables. Iteration is truncated when the flow field distribution in the new approximation differs from that of the last approximation within admissible degree of error. This algorithm is straightforward for subcritical flows where $\Delta_{d i}(x) \geq 0$ for all $x$; however, it needs to be supplemented by the normal shock relations and by the location of the normal shock (shock fitting) for supercritical flows. Without entering into the difficult analysis of the supercritical flow solution, we attempt to set up a criterion that can predict whether or not for given initial reservoir conditions, nozzle geometry and $Q_{i n}^{\prime}(x)$ the flow is supercritical. We restrict our discussion to supersonic heat addition $\left(x_{b}>0\right)$ because subsonic heat addition $\left(x_{b}<0\right)$ requires the influence of the exit boundary conditions as well [for numerical solution of the differential theory with heat addition in the subsonic region see Jungclaus and van Raay (1967)]. It follows at once from Proposition 2.2.2 that the flow is subcritical if

$$
\frac{Q(x)}{c_{p 0} T_{0}}<\frac{Q_{I I}^{*}(x)}{c_{p 0} T_{0}}
$$

for all $x>x_{b}>0$. If inequality (25) is violated for some $x>x_{b}>0$, it appears that, although $Q_{I I}^{*}(x)$ has been exceeded, $Q(x)$ may still remain less than $Q^{*}(z)$, thus no conclusion can be reached. In this case the flow may be subcritical or supercritical. In order to be able to gain an insight to the resolution of this problem, we first define the point $x^{*}$ implicitly by $\Delta\left(x^{*}\right) \equiv 0$ which is equivalent to $Q^{*}\left(x^{*}\right) /\left(c_{p 0} T_{0}\right) \equiv Q\left(x^{*}\right) /\left(c_{p 0} T_{0}\right)$ (critical flow condition). We also let $x_{1}^{*}$ and $x_{I I}^{*}$ be predictions of $x^{*}$ in Model I and Model II respectively. They can be directly evaluated from

$$
\frac{Q\left(x_{j}^{*}\right)}{c_{p 0} T_{0}}=\frac{Q_{j}^{*}\left(x_{j}^{*}\right)}{c_{p 0} T_{0}} \quad j=I, I I
$$

where $Q_{j}^{*}(x) /\left(c_{p 0} T_{0}\right) j=I, I I$ are given by Eqs. (21) and (22). It follows immediately from Proposition 2.2.2 that

$$
0<x_{b}<x_{I}^{*}<x_{I I}^{*}<x^{*} .
$$


Figure 3

Possible predictions of the critical amount of heat as a function of the axial coordinate in different models for diabatic nozzle flows.

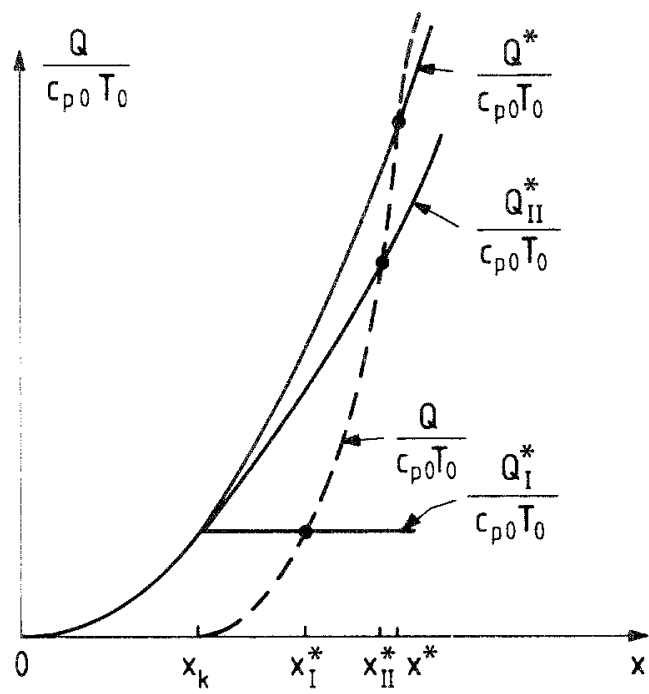

We now suppose that $Q(x)>Q_{I I}^{*}(x)$ for some $x>x_{I I}^{*}>x_{I}^{*}>x_{b}>0$ and try to determine whether or not the flow is supercritical. Although a definite answer seems not possible in this case, the derivative of $Q(x)$ may be helpful in obtaining some information. If $d Q / d x=O(1)$ numerically at $x=x_{I I}^{*}$ and within a distance of $O\left(x_{I I}^{*}-x_{b}\right)$ downstream, then $Q(x)$ will intersect $Q^{*}(x)$ at $x^{*}>x_{I I}^{*}$ and the flow will be supercritical (Fig. 3). Otherwise the flow may remain subcritical or may become supercritical with a weak normal shock wave (such a flow may be called a flow bordering on supercritical regime or a flow in the transition regime). A more definitive criterion requires the evaluation of $Q^{*}(x)$ given by Eq. (20), which requires the exact evaluation of $R_{d i}(x)$ and which can only be achieved by iteration.

\subsection{Nozzle flows with nonequilibrium condensation}

For nozzle flows with nonequilibrium condensation we set $Q_{\text {in }}^{\prime}(x) \equiv 0$ in Eq. (5) and retain the notation and normalization of Eqs. (1)-(4). As mentioned earlier Eqs. (1)-(4) do not constitute a complete system in this case unless the nonequilibrium condensation rate equation for $g^{\prime}$ is considered. In order to complete the system we assume that $g^{\prime} \equiv 0$ for $x \leq x_{s}$ and $g^{\prime}=g^{\prime}(x)$ is some arbitrary, positive, strictly increasing function for $x>x_{s}$. The functions $R\left(g^{\prime}, x\right)$ and $\Delta\left(g^{\prime}, x\right)$ for these flows are then respectively given by Eqs. (6) and (8) with

$$
\frac{q\left(g^{\prime}, x\right)}{c_{p 0} T_{0}} \equiv \frac{g^{\prime} L^{\prime}(T)}{c_{p 0}^{\prime} T_{0}^{\prime}}
$$


The flow field for $x \leq x_{s}$ where $g^{\prime} \equiv 0$ is given by the classical isentropic nozzle flow solution discussion in Section 2.1. Due to the existence of a nonequilibrium variable (the condensate mass fraction $g^{\prime}$ ), isentropic flows will be termed frozen flows in this section and will be denoted by subscript $f$. Thus for $x \leq x_{s}$ we have $R\left(g^{\prime}, x\right)=R_{f}(x) \equiv R_{i s}(x)$ and $\Delta\left(g^{\prime}, x\right)=$ $\Delta_{f}(x) \equiv \Delta_{i s}(x)$ which are respectively given by Eqs. (12) and (13). To discuss the flow field for $x \geq x_{s}$, we first define the onset point $x_{k}$ as the location of the collapse of the supersaturated vapor state (which, for example, can be determined empirically by static pressure measurements within the precision achieved) and from now on reserve the subscript $k$ for variables at the onset point. Before proceeding any further, we first discuss the nature of flow in the interval $x_{s} \leq x \leq x_{k}$. From the asymptotic theories of condensation [Blythe and Shih (1976) and Clarke and Delale (1986)], one can easily show that in the interval $x_{s} \leq x \leq x_{k}$ the condensate mass fraction $g^{\prime}$ is exponentially small everywhere except for a thin zone near $x_{k}$. In both theories in the interval $x_{s} \leq x \leq x_{k}$ the nearly frozen approximation $R\left(g^{\prime}, x\right)=R_{f}(x), L^{\prime}(T)=L^{\prime}\left(T_{f}\right)$ is easily verified. Experimentally it is almost impossible to distinguish between $R\left(g^{\prime}, x\right)$ and $R_{f}(x)$, $L^{\prime}(T)$ and $L^{\prime}\left(T_{f}\right)$ in the interval $x_{s} \leq x \leq x_{k}$. Numerical computations [e.g. see Schnerr and Dohrmann (1989)] also support the same hypothesis. Thus we can without hesitation take $R\left(g^{\prime}, x\right)=R_{f}(x)$ in the interval $x_{s} \leq x \leq x_{k}$. In particular at $x=x_{k}$ we have

$$
\begin{aligned}
R_{k} & \equiv R\left(g_{k}^{\prime}, x_{k}\right) \approx R_{f}\left(x_{k}\right) \\
& =\frac{M_{s}}{M_{k}} \sqrt{\frac{1+\frac{(\gamma-1)}{2} M_{s}^{2}}{1+\frac{(\gamma-1)}{2} M_{k}^{2}}}\left(1+\gamma M_{k}^{2}\right)-\gamma M_{s}^{2}-1
\end{aligned}
$$

where $g_{k}^{\prime}$ and $M_{k}$ denote respectively the condensate mass fraction $(\ll 1)$ and the local frozen Mach number $(>1)$ at onset. Downstream of the onset point $\left(x>x_{k}\right)$ the nearly frozen approximation is erroneous since $g^{\prime}$ grows in magnitude. Even if $g^{\prime}(x)$ were known downstream, the integroalgebraic system (7)-(11) would not explicitly determine the flow field unless $R\left(g^{\prime}, x\right)$ and $L^{\prime}(T)$ are simultaneously evaluated downstream. On the other hand one may exceed the critical amount of heat downstream of $x_{k}$. It follows immediately from Eq. (8) that for a continuous solution the critical amount of heat at any location $x$, herein denoted by $q^{*}\left(g^{\prime}, x\right)$, not to be exceeded by heat addition from condensation is defined by

$$
\frac{q^{*}\left(g^{\prime}, x\right)}{c_{p 0} T_{0}} \equiv \frac{\left(\left[1+u_{s}^{2}+R\left(g^{\prime}, x\right)\right] /\left(2 u_{s}\right)\right)^{2}}{T_{0} \Theta\left(g^{\prime}\right)}-1
$$


where $\Theta\left(g^{\prime}\right)$ is defined by

$$
\Theta\left(g^{\prime}\right) \equiv \frac{\left(1+\gamma+(\gamma-1) \frac{\mu_{0}}{\mu_{v}} g^{\prime}\right)\left(1-\frac{\mu_{0}}{\mu_{v}} g^{\prime}\right)}{2 \gamma} .
$$

Similar to the definition in the preceding section for diabatic nozzle flows, we call a nozzle flow with nonequilibrium condensation subcritical if

$$
\frac{q\left(g^{\prime}, x\right)}{c_{p 0} T_{0}}<\frac{q^{*}\left(g^{\prime}, x\right)}{c_{p 0} T_{0}}
$$

for all $x>x_{k}$ and supercritical if

$$
\frac{q\left(g^{\prime}, x\right)}{c_{p 0} T_{0}}>\frac{q^{*}\left(g^{\prime}, x\right)}{c_{p 0} T_{0}}
$$

for some $x>x_{k}$. In order to be able to discuss the flow downstream of $x_{k}$ any further and find out whether the flow has become supercritical or not, we introduce some models which approximate the actual flow field downstream of $x_{k}$ for given $g^{\prime}=g^{\prime}(x)$ and $L^{\prime}=L^{\prime}(T)$ :

Model 1 [Wegener and Mack (1958)]. This model is defined by assuming that $R\left(g^{\prime}, x\right)=R_{k} \approx R_{f}\left(x_{k}\right)$ and $\Theta\left(g^{\prime}\right)=\Theta(0)=(\gamma+1) /(2 \gamma)$ for $x>x_{k}$. Such flows can be realized only approximately when $0<g^{\prime}<\omega_{0} \ll 1$ and $0<d A / d x \ll 1$ for $x>x_{k}$. In particular the function $\Delta$, denoted by $\Delta_{1}\left(g^{\prime}, x\right)$ in this model, and the critical amount of heat $q^{*}$, denoted by $q_{1}^{*}\left(g^{\prime}, x\right)$ in this model, evaluate to

$$
\begin{aligned}
\Delta_{1}\left(g^{\prime}, x\right)= & \frac{\left(1+\frac{(\gamma-1)}{2} M_{s}^{2}\right)}{4 \gamma M_{k}^{2}\left(1+\frac{(\gamma-1)}{2} M_{k}^{2}\right)} \\
& \times\left(\left(M_{k}^{2}-1\right)^{2}-2(\gamma+1) M_{k}^{2}\left[1+\frac{(\gamma-1)}{2} M_{k}^{2}\right] \frac{q\left(g^{\prime}, x\right)}{c_{p 0} T_{0}}\right) \\
< & \Delta\left(g^{\prime}, x\right) \\
\frac{q_{1}^{*}\left(g^{\prime}, x\right)}{c_{p 0} T_{0}}= & \frac{\left(M_{k}^{2}-1\right)^{2}}{2(\gamma+1) M_{k}^{2}\left(1+\frac{(\gamma-1)}{2} M_{k}^{2}\right)}<\frac{q^{*}\left(g^{\prime}, x\right)}{c_{p 0} T_{0}}
\end{aligned}
$$

for $x>x_{k}$ where $q\left(g^{\prime}, x\right) /\left(c_{p 0} T_{0}\right)$ is given by Eq. (27).

Model 2. In this model we take $R=R_{f}(x) \equiv R_{i s}(x)$ and $\Theta\left(g^{\prime}\right)=$ $\Theta(0)=(\gamma+1) /(2 \gamma)$ for $x>x_{k}$ where $R_{i s}(x)$ is given by Eq. (12) with subscript $b$ replaced by subscript $s$ and $M(x)$ replaced by $M_{f}(x)$, which 
denotes the local frozen (at $g^{\prime}=0$ ) Mach number. Such flows can not in principle be realized physically since heat addition from nonequilibrium condensation without increase of entropy is impossible, therefore predictions of this model are to be used only as approximations to the actual flow field. The function $\Delta$, denoted by $\Delta_{2}\left(g^{\prime}, x\right)$ in this model, and the critical amount of heat $q^{*}$, denoted by $q_{2}^{*}\left(g^{\prime}, x\right)$ in this model, are given respectively by Eqs. (31) and (32) where $M_{k}$ is to be replaced by $M_{f}(x)$ for $x>x_{k}$.

Model 3. This model is defined by simply taking $R\left(g^{\prime}, x\right)=R_{k} \approx R_{f}\left(x_{k}\right)$ for $x>x_{k}$, a condition which is also assumed in defining Model 1. This model can be approximately realized for flows where $0<d A / d x \ll 1$ for $x>x_{k}$. In fact it can be shown that this model is precisely what is achieved in the differently scaled droplet growth zones of the asymptotic theories of Blythe and Shih (1976) and Clarke and Delale (1986). In this case the function $\Delta$, denoted herein by $\Delta_{3}\left(g^{\prime}, x\right)$, and the critical amount of heat $q^{*}$, denoted herein by $q_{3}^{*}\left(g^{\prime}, x\right)$, are given by

$$
\begin{aligned}
\Delta_{3}\left(g^{\prime}, x\right)= & \frac{\left(1+\frac{(\gamma-1)}{2} M_{s}^{2}\right)}{4 \gamma M_{k}^{2}\left(1+\frac{(\gamma-1)}{2} M_{k}^{2}\right)} \\
& \times\left(\left(1+\gamma M_{k}^{2}\right)^{2}-4 \gamma M_{k}^{2}\left[1+\frac{(\gamma-1)}{2} M_{k}^{2}\right] \Theta\left(g^{\prime}\right)\right. \\
& \left.\times\left[1+\frac{q\left(g^{\prime}, x\right)}{c_{p 0} T_{0}}\right]\right) \\
& <\Delta\left(g^{\prime}, x\right) \\
\frac{q_{3}^{*}(x)}{c_{p 0} T_{0}}= & \frac{1-2\left[\left(1-\frac{\mu_{0}}{\mu_{v}} g^{\prime}\right)^{2}-\gamma\left(\frac{\mu_{0}}{\mu_{v}} g^{\prime}\right)^{2}\right] M_{k}^{2}+\left[1+(\gamma-1)\left(\frac{\mu_{0}}{\mu_{v}} g^{\prime}\right)\right]^{2} M_{k}^{4}}{2 M_{k}^{2}\left[1+\frac{(\gamma-1)}{2} M_{k}^{2}\right]\left[\gamma+1-2\left(\frac{\mu_{0}}{\mu_{v}} g^{\prime}\right)-(\gamma-1)\left(\frac{\mu_{0}}{\mu_{v}} g^{\prime}\right)^{2}\right]} \\
< & \frac{q^{*}\left(g^{\prime}, x\right)}{c_{p 0} T_{0}}
\end{aligned}
$$

for $x>x_{k}$.

Model 4. In this model we take $R\left(g^{\prime}, x\right)=R_{f}(x)$ for $x>x_{k}$ (this condition is also used to define Model 2). This model can not be realized physically either since heat addition from nonequilibrium condensation without increase of entropy is impossible. Therefore it should only be used for approximate predictions. In this model the function $\Delta$, herein denoted by $\Delta_{4}\left(g^{\prime}, x\right)$, and the critical amount of heat $q^{*}$, herein denoted by 
$q_{4}^{*}\left(g^{\prime}, x\right)$, are precisely given by Eqs. (33) and (34) where the onset Mach number $M_{k}$ should be replaced by the local frozen Mach number $M_{f}(x)$.

The following results which can be used to compare the above models can easily be proved.

Proposition 2.3.1. For $x>x_{k}>0, \Theta\left(g^{\prime}\right)$ is strictly decreasing and $R\left(g^{\prime}, x\right)$ is strictly increasing, thereby $q^{*}\left(g^{\prime}, x\right)$ and $q_{j}^{*}\left(g^{\prime}, x\right) j=2,3,4$ are strictly increasing whereas $q_{1}^{*}\left(g^{\prime}, x\right)$ is a constant.

Proposition 2.3.2. At any location $x>x_{k}>0$ where $g^{\prime}=g^{\prime}(x)$ we have

$$
\Delta_{1}\left(g^{\prime}, x\right)<\Delta_{2}\left(g^{\prime}, x\right)<\Delta_{4}\left(g^{\prime}, x\right)<\Delta\left(g^{\prime}, x\right)
$$

and

$$
\Delta_{1}\left(g^{\prime}, x\right)<\Delta_{3}\left(g^{\prime}, x\right)<\Delta_{4}\left(g^{\prime}, x\right)<\Delta\left(g^{\prime}, x\right),
$$

or equivalently

$$
q_{1}^{*}\left(g^{\prime}, x\right)<q_{2}^{*}\left(g^{\prime}, x\right)<q_{4}^{*}\left(g^{\prime}, x\right)<q^{*}\left(g^{\prime}, x\right)
$$

and

$$
q_{1}^{*}\left(g^{\prime}, x\right)<q_{3}^{*}\left(g^{\prime}, x\right)<q_{4}^{*}\left(g^{\prime}, x\right)<q^{*}\left(g^{\prime}, x\right) .
$$

It follows directly from Proposition 2.3.2 that among all modes discussed Model 4 seems to predict the best approximation to the actual flow provided that $g^{\prime}(x)$ and $L^{\prime}(T)$ are known. As a first step $L^{\prime}(T)$ can be evaluated from its frozen value $L^{\prime}\left(T_{f}\right)$ and then can be corrected for from the solution for $T$ by Eq. (11). Although no further comment on the rate equation will be made, it suffices to mention that for any given nucleation and droplet growth theories (in other words for any given working fluid) the function $g^{\prime}=g^{\prime}(x)$ has to be solved from the nonequilibrium integral rate equation constructed by substituting for the thermodynamic state variables from Eqs. (7) -(11) [asymptotic solutions with different ordering of the double-limit process are already given by Blythe and Shih (1976) and Clarke and Delale (1986)]. The actual subcritical flow solution can in principle be obtained from this solution by iteration similar to the procedure discussed in detail for diabatic flows. The solution for supercritical flows can also be given by supplementing the subcritical flow solution by normal shock relations and shock fitting. Without going into further details we now attempt to find out if the flow becomes supercritical or not. For this reason we suppose that the critical flow condition

$$
\frac{q^{*}\left(g^{\prime}, x\right)}{c_{p 0} T_{0}}=\frac{q\left(g^{\prime}, x\right)}{c_{p 0} T_{0}}
$$


is reached at some location $x^{*}$ in the nozzle where the condensate mass fraction has the value $g^{*} \equiv g^{\prime}\left(x^{*}\right)$. It is then obvious that for given initial reservoir conditions, nozzle geometry and working fluid, the flow is subcritical if $g^{\prime}(x)<g^{*}$ for all $x>x_{k}>0$ and supercritical if $g^{\prime}(x)>g^{*}$ for some $x>x_{k}>0$. In particular for the flow of a mixture of condensible vapor with carrier gas (e.g. the expansion of moist air) if we assume that all of the vapor has condensed, we arrive at the criterion that the flow is subcritical whenever $\omega_{0}<g^{*}$ and supercritical if $\omega_{0}>g^{*}$. On the other hand under given flow conditions and geometry $g^{*}$ yields the largest possible value of the condensate mass fraction at any location for the subcritical flow of a pure vapor. Therefore the prediction of $g^{*}$ is of vital importance in determining whether or not the flow is supercritical. Since exact evaluation of $g^{*}$ seems not possible at this stage, we discuss predictions for it in the models introduced above, if possible. We let $g_{j}^{*}$ $j=1,2,3,4$ be the prediction for $g^{*}$ in the $j$ th Model and $x_{j}^{*} j=1,2$, 3,4 be the corresponding location in nozzle where the critical flow condition in the $j$ th Model is reached. It then follows from Proposition 2.3.2 that

$$
x_{1}^{*}<x_{2}^{*}<x_{4}^{*}<x^{*}
$$

and

$$
x_{1}^{*}<x_{3}^{*}<x_{4}^{*}<x^{*},
$$

or equivalently

$$
g_{1}^{*}<g_{2}^{*}<g_{4}^{*}<g^{*},
$$

and

$$
g_{1}^{*}<g_{3}^{*}<g_{4}^{*}<g^{*} .
$$

Figure 4 shows a typical ordering of $x^{*}$ and $x_{j}^{*} j=1,2,3,4$. Although Model 4 seems to yield the best approximation for the prediction of $g^{*}$, it turns out that only Model 3 and Model 1 yield quantitative results whereas Model 4 and Model 2 can be used to interpret how well these quantitative results approximate $g^{*}$. For this reason we first discuss the prediction of Model 3 in detail. We define $z$ and $\alpha$ by

$$
\begin{aligned}
& z \equiv \frac{\mu_{0}}{\mu_{v}} g_{3}^{*} \\
& \alpha \equiv \frac{\mu_{v} L_{3}^{*}}{\mu_{0} c_{p 0}^{\prime} T_{0}^{\prime}}
\end{aligned}
$$




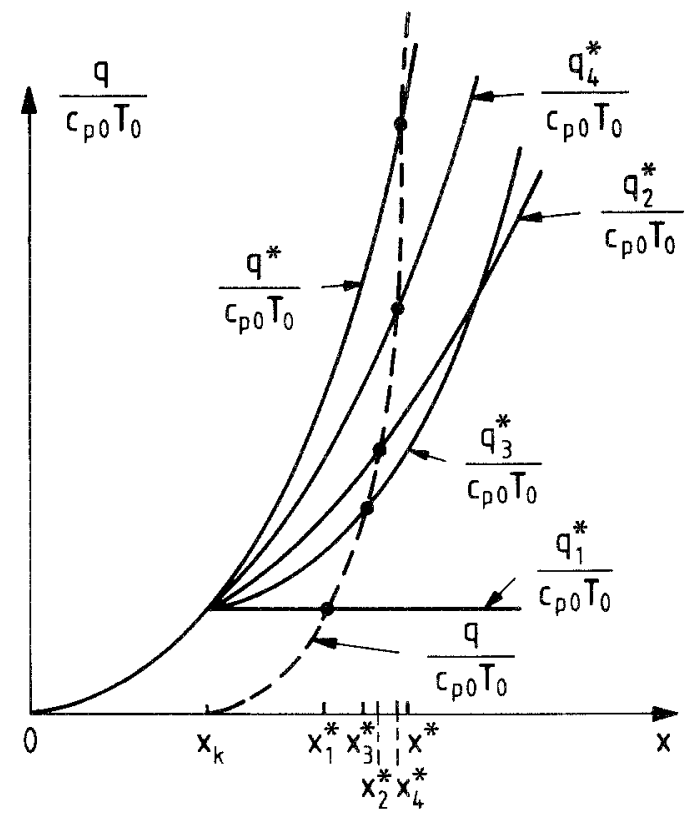

Figure 4

Possible predictions of the critical amount of heat as a function of the axial coordinate in different models for nozzle flows with nonequilibrium condensation.

where $L_{3}^{*} \equiv L^{\prime}\left[T\left(g_{3}^{*}, x_{3}^{*}\right)\right]$. From the critical condition, Eq. (35) in Model 3, we obtain at once a cubic equation to be solved for $z$ :

$$
z^{3}+A_{2} z^{2}+A_{1} z+A_{0}=0
$$

where

$$
\begin{aligned}
& A_{2} \equiv \frac{2 \alpha+(\gamma-1)}{\alpha(\gamma-1)}, \\
& A_{1} \equiv-\frac{[(\gamma+1) \alpha-2]}{\alpha(\gamma-1)},
\end{aligned}
$$

and

$$
A_{0} \equiv \frac{\left(M_{k}^{2}-1\right)^{2}}{2 \alpha(\gamma-1) M_{k}^{2}\left(1+\frac{(\gamma-1)}{2} M_{k}^{2}\right)} .
$$

Equation (38) evaluates $g_{3}^{*}$ as a function of the onset Mach number $M_{k}$ provided that $L_{3}^{*}$ is known. The branch of the cubic equation (38) corresponding to the physical solution for $g_{3}^{*}$ must satisfy the condition $g_{3}^{*} \rightarrow 0$ as $M_{k} \rightarrow 1$. It is remarkable that Eq. (38) could also be arrived at using the solution of the droplet growth zones in the asymptotic theories of Blythe and Shih (1976) and Clarke and Delale (1986). Before we discuss how to 
evaluate $M_{k}$ and $L_{3}^{*}$ for given initial reservoir conditions, nozzle geometry and working fluid, we would like to check how good an estimate $g_{3}^{*}$ is for $g^{*}$. For this reason we define $\delta \equiv x_{3}^{*}-x_{k}$ where $x_{3}^{*}$ is given by $g_{3}^{*}=g^{\prime}\left(x_{3}^{*}\right)$ (in asymptotic theories $\delta$ is simply of the order of magnitude of the normalized thickness of the droplet growth zone). It is clear from Proposition 2.3.2 that $\Delta\left(g_{3}^{*}, x_{3}^{*}\right)>0$. Actually it can be shown that $\Delta\left(g_{3}^{*}, x_{3}^{*}\right)=|O(\delta)|$. In particular when $\delta \rightarrow 0, g_{3}^{*} \rightarrow g^{*}$. We therefore arrive at the conclusion that for all practical purposes $g_{3}^{*}$ yields a good aproximation to $g^{*}$ whenever $\delta \ll 1$. An estimate of $\delta$ requires the solution of the condensation rate equation. For example experiments and numerical computations by Schnerr (1989) and Schnerr and Dohrmann (1989) show that $\delta$ ranges between 0.2 and 0.6 numerically for different nozzle geometries (for estimates of $\delta$ in asymptotic theories see Blythe and Shih and Clarke and Delale). Thus $\delta$ is small compared to unity, but not too small to be ignored. This means that $g_{3}^{*}$ differs from $g^{*}$, but not considerably. On the other hand if Model 3 is replaced by Model 1, we obtain the results of Wegener and Mack (1958):

$$
g_{1}^{*}=\frac{c_{p 0}^{\prime} T_{0}^{\prime}}{L_{1}^{*}} \frac{\left(M_{k}^{2}-1\right)^{2}}{2(\gamma+1) M_{k}^{2}\left[1+\frac{(\gamma-1)}{2} M_{k}^{2}\right]}
$$

where $L_{1}^{*} \equiv L^{\prime}\left[T\left(g_{1}^{*}, x_{1}^{*}\right)\right]$. Figures 5-7 show typical solutions of $g_{3}^{*}$ and $g_{1}^{*}$, given respectively by Eqs. (38) and (42), as a function of the onset Mach number $M_{k}$ for the expansion of moist air in different nozzles whose geometric specifications are listed in Table 1. In particular Fig. 5 shows the effect of the variation of the latent heat on the solution of Eqs. (38) and (42). As can be clearly seen (at least for the expansion of moist air) the predictions with the minimum latent heat yield only slightly higher values of $g_{3}^{*}$ and $g_{1}^{*}$ than those with the maximum latent heat in the operational range of the nozzle. Therefore for all practical computations we set without hesitation

$$
L_{1}^{*}=L_{3}^{*}=L_{\min }^{\prime}
$$

where $L_{\min }^{\prime}$ is the minimum value of the latent heat in the operational range

Table 1

Geometric specifications of the circular arc nozzles used in Figs. 5-8.

\begin{tabular}{llll}
\hline Nozzle & $\begin{array}{l}\text { Throat height } \\
2 y^{*}[\mathrm{~mm}]\end{array}$ & $\begin{array}{l}\text { Circular nozzle } \\
\text { radius } R^{*}[\mathrm{~mm}]\end{array}$ & $\begin{array}{l}\text { Experimental } \\
\text { source }\end{array}$ \\
\hline Nozzle \#1 & 30 & 400 & Schnerr (1986) \\
Nozzle \#2 & 60 & 584 & Barschdorff (1967) \\
Nozzle \#3 & 40 & 127 & Wegener and Pouring (1958) \\
\hline
\end{tabular}



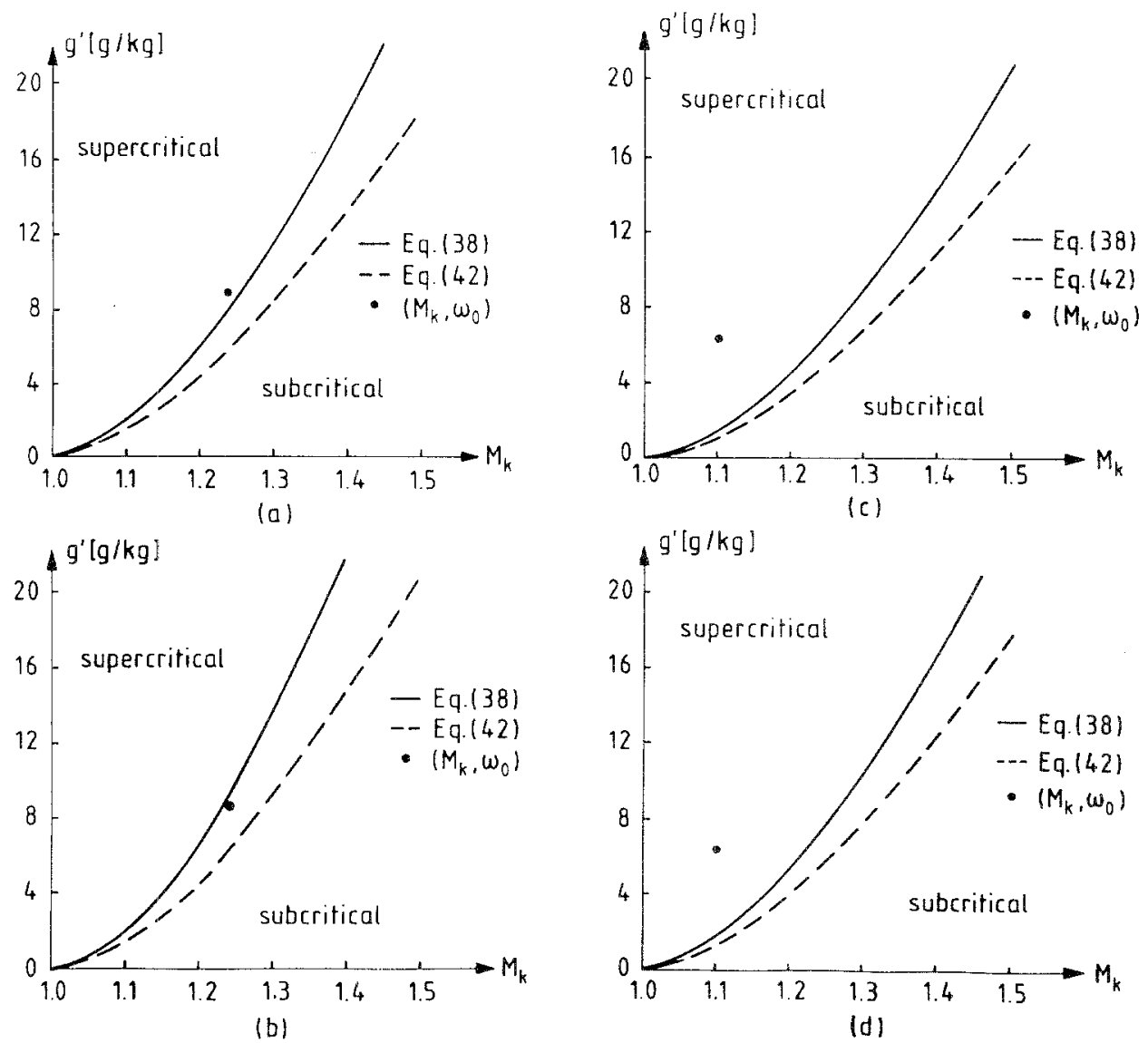

Figure 5

The effect of latent heat variation on the predictions of Eqs. (38) and (42) in subcritical and supercritical expansion of moist air through Nozzle \#2 used by Barschdorff (1967) in experiments conducted by Schnerr (1986). (a) subcritical flow with $L^{\prime}=2839 \mathrm{~kJ} / \mathrm{kg}\left(\phi_{0}=34.3 \%, \omega_{0}=8.9 \mathrm{~g} / \mathrm{kg}\right.$, $\left.T_{0}^{\prime}=302.4^{\circ} \mathrm{K} \Rightarrow M_{k} \approx 1.24\right)$, (b) subcritical flow with $L^{\prime}=2501 \mathrm{~kJ} / \mathrm{kg} \quad\left(\phi_{0}=34.3 \%, \omega_{0}=8.9 \mathrm{~g} / \mathrm{kg}\right.$, $\left.T_{0}^{\prime}=302.4^{\circ} \mathrm{K} \Rightarrow M_{k} \approx 1.24\right)$, (c) supercritical flow with $L^{\prime}=2839 \mathrm{~kJ} / \mathrm{kg}\left(\phi_{0}=60.9 \%, \omega_{0}=6.3 \mathrm{~g} / \mathrm{kg}\right.$, $\left.T_{0}^{\prime}=287.6^{\circ} \mathrm{K} \Rightarrow M_{k} \approx 1.1\right)$, (d) supercritical flow with $L^{\prime}=2501 \mathrm{~kJ} / \mathrm{kg}\left(\phi_{0}=60.9 \%, \omega_{0}=6.3 \mathrm{~g} / \mathrm{kg}\right.$, $T_{0}^{\prime}=287.6^{\circ} \mathrm{K} \Rightarrow M_{k} \approx 1.1$ ).

of the nozzle. Consequently in Figs. 6 and 7, $L_{1}^{*}$ and $L_{3}^{*}$ are evaluated by Eq. (43). In all of the figures at any onset Mach number $M_{k}$ we have $g_{1}^{*}<g_{3}^{*}$ in agreement with Proposition 2.3.2. For the specified initial relative humidity and nozzle geometry the onset Mach number $M_{k}$ is calculated by the Zierep-Lin (1967) similarity law using empirical values for the exponents. Under specified initial reservoir conditions and nozzle geometry, Figs. 5(b), 6(a), and $7(\mathrm{a})^{1}$ show that at the onset Mach number

Since Nozzle 3 in Table 1 is effectively two-dimensional, the predictions in Fig. 7 are understood for the flow along the centerline. 

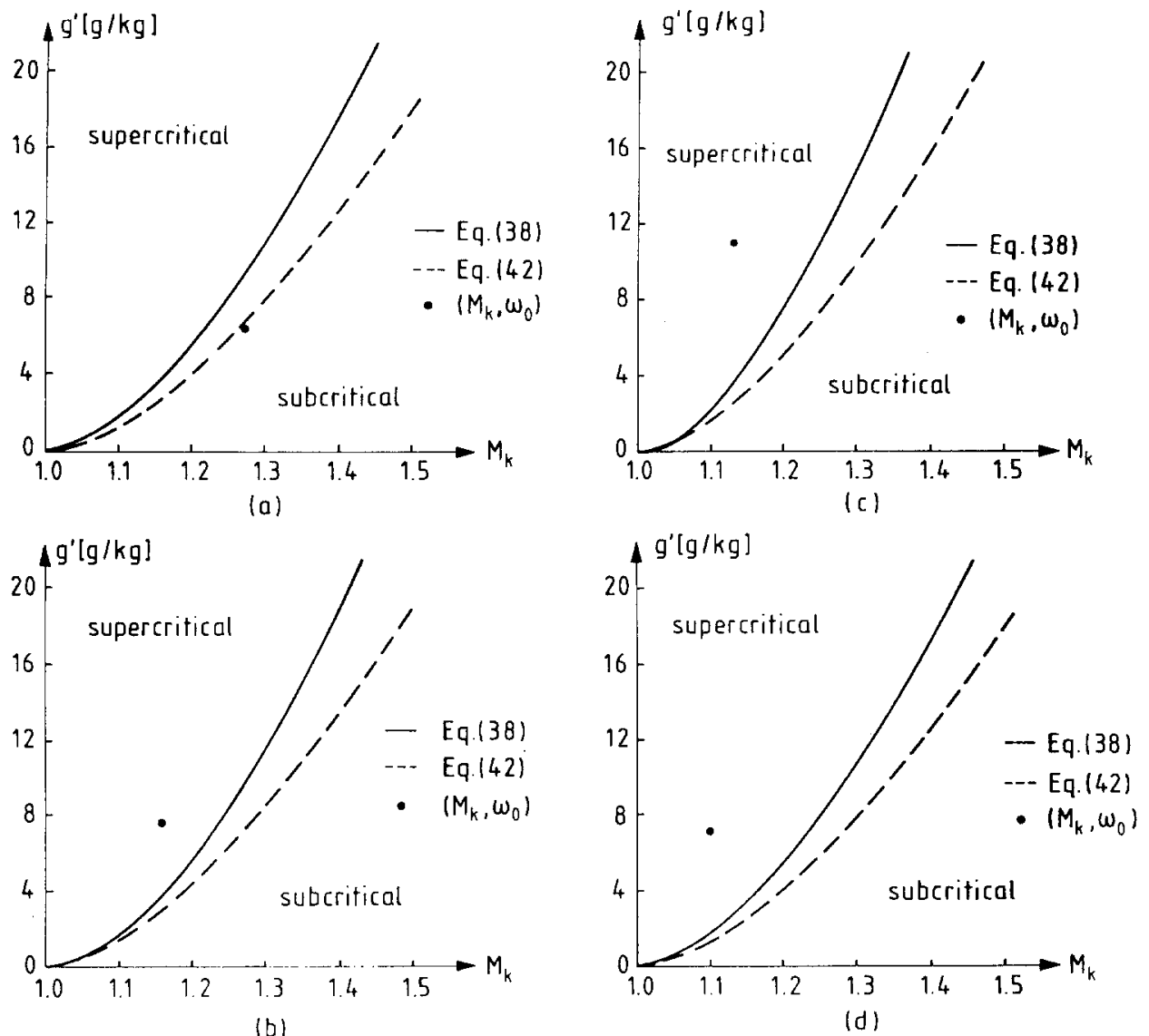

Figure 6

Predictions of Eqs. (38) and (42) with $L^{\prime}=2501 \mathrm{~kJ} / \mathrm{kg}$ for the expansion of moist air through Nozzle \# 1 in experiments conducted by Schnerr (1986). (a) subcritical flow $\left(\phi_{0}=38.0 \%, \omega_{0}=6.5 \mathrm{~g} / \mathrm{kg}, T_{0}^{\prime}=\right.$ $295.6^{\circ} \mathrm{K} \Rightarrow M_{k} \approx 1.27$ ), (b) supercritical flow $\left(\phi_{0}=59.4 \%, \omega_{0}=7.6 \mathrm{~g} / \mathrm{kg}, T_{0}^{\prime}=290.8^{\circ} \mathrm{K} \Rightarrow M_{k} \approx 1.16\right.$ ), (c) supercritical flow $\left(\phi_{0}=65.2 \%, \omega_{0}=11.0 \mathrm{~g} / \mathrm{kg}, T_{0}^{\prime}=295.8^{\circ} \mathrm{K} \Rightarrow M_{k} \approx 1.13\right)$, (d) supercritical flow $\left(\phi_{0}=76.4 \%, \omega_{0}=7.0 \mathrm{~g} / \mathrm{kg}, T_{0}^{\prime}=285.7^{\circ} \mathrm{K} \Rightarrow M_{k} \approx 1.1\right)$.

$M_{k}$ of the particular subcritical experimental run, $\omega_{0}$ always lies below the curve estimated by Eq. (38) whereas it is bordering on the curve estimated by Eq. (42). On the other hand in Figs. 5(d), 6(b)-(d) and 7(b) at the onset Mach number $M_{k}$ of the particular supercritical experimental run, $\omega_{0}$ lies much above both curves estimated by Eqs. (38) and (42). In particular the schlieren photographs of Fig. 8 show complete agreement with the results shown in Fig. 6 for the particular subcritical and supercritical experimental runs. The above consideration for the expansion of moist air demonstrates the following criterion: 
Figure 7

Predictions of Eqs. (38) and (42) with $L^{\prime}=2501 \mathrm{~kJ} /$ $\mathrm{kg}$ for the expansion of moist air through Nozzle \#3 in experiments conducted by Schnerr (1986) using nozzle geometry of Wegener and Pouring (1964). (a) subcritical flow $\left(\phi_{0}=32.8 \%, \omega_{0}=8.5 \mathrm{~g} /\right.$ $\mathrm{kg}, \quad T_{0}^{\prime}=302.4^{\circ} \mathrm{K} \Rightarrow M_{k} \approx 1.3$ ), (b) supercritical flow $\left(\phi_{0}=64.0 \%, \omega_{0}=9.0 \mathrm{~g} / \mathrm{kg}, T_{0}^{\prime}=292.1^{\circ} \mathrm{K} \Rightarrow\right.$ $\left.M_{k} \approx 1.13\right)$.
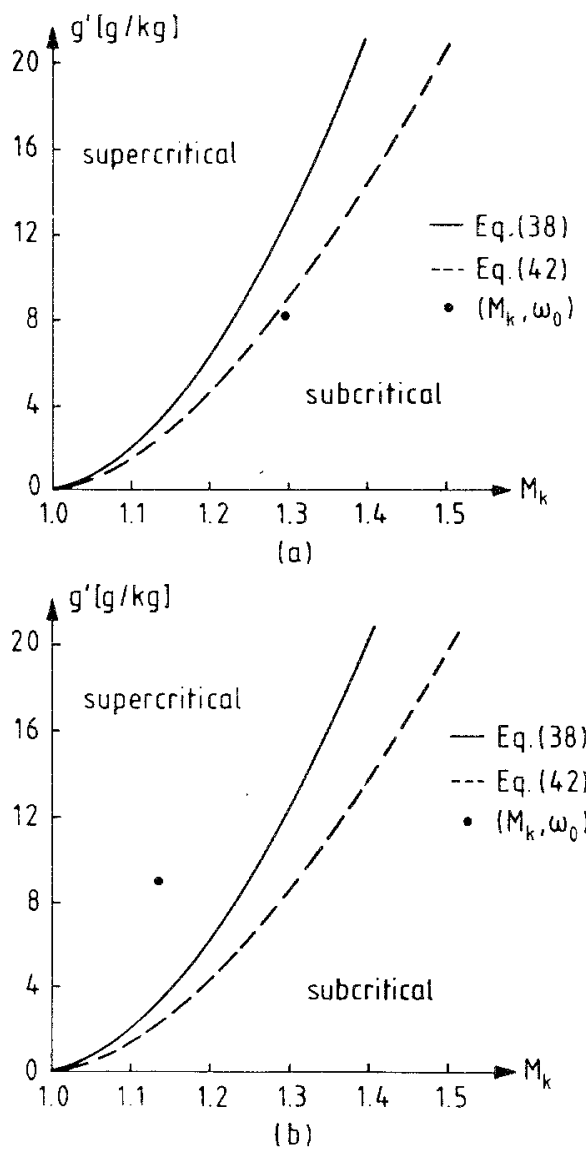

For the expansion of a mixture of condensible vapor with a carrier gas on the assumption that all of the vapor has condensed

(i) the flow is definitely subcritical if $\omega_{0}<g_{3}^{*}$,

(ii) the flow is supercritical if $\omega_{0}$ is considerably greater than $g_{3}^{*}$, i.e $\left(\omega_{0}-g_{3}^{*}\right) / \omega_{0}=O(1)$, and

(iii) the flow is bordering on supercritical flow if $\omega_{0}$ is only slightly greater than $g_{3}^{*}$, i.e. $\left(\omega_{0}-g_{3}^{*}\right) / \omega_{0}=o(1)$.

For the expansion of pure vapor $\left(\omega_{0}=1\right) g_{3}^{*}$ is a lower bound for the maximum of the condensate mass fraction that can be attained in subcritical flows.

Now that we have the predictions $g_{3}^{*}$ and $g_{1}^{*}$ for $g^{*}$ given explicitly by Eqs. (38) and (42), we attempt to evaluate the predictions $g_{4}^{*}$ and $g_{2}^{*}$ for $g^{*}$. It follows immediately from the critical flow conditions in Model 4 and 

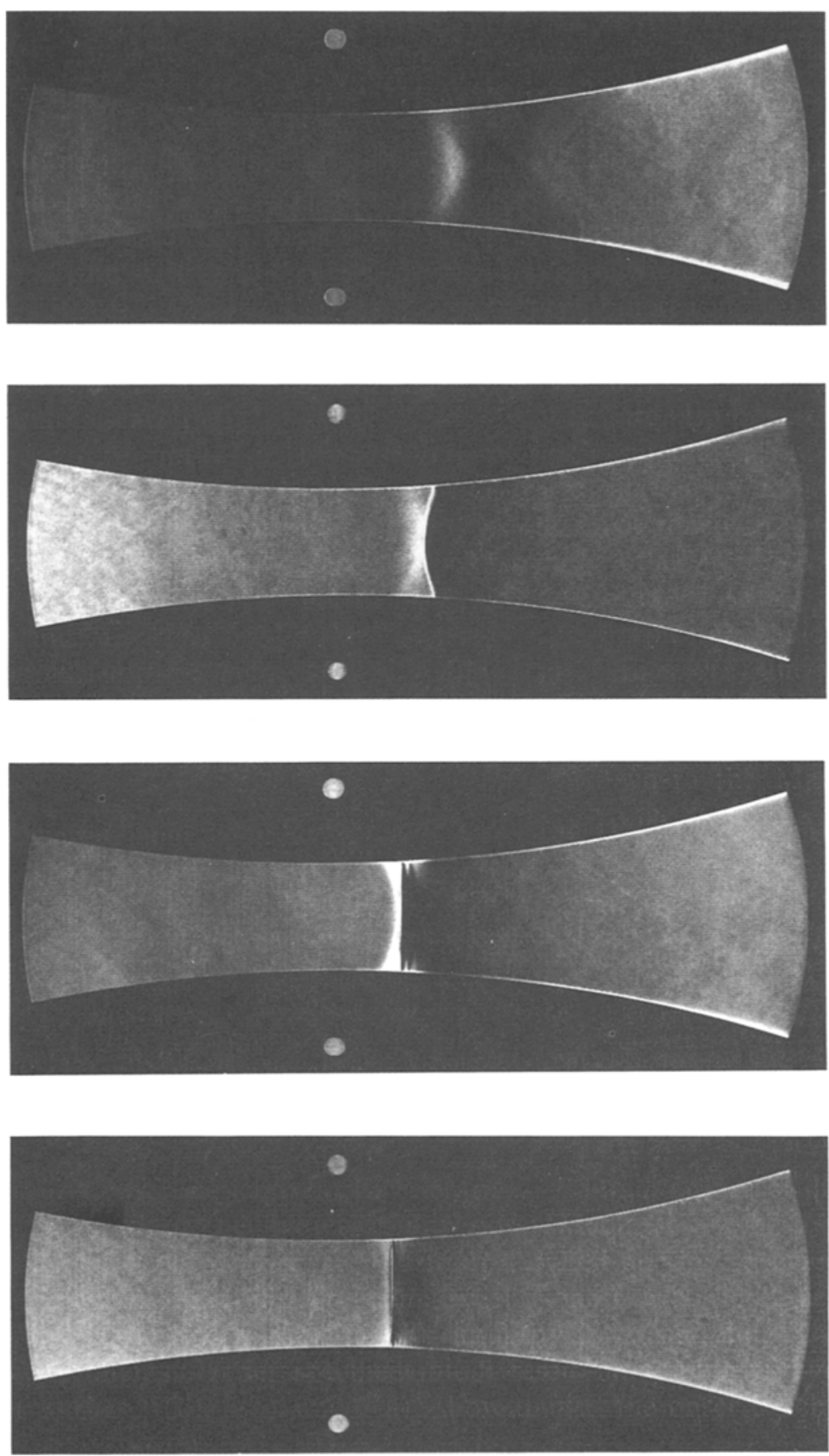

Figure 8

Flow visualization by schlieren photographs of experimental runs conducted by Schnerr (1986) corresponding to data given in Fig. 6(a)-(d). 
Model 1 that $g_{4}^{*}$ satisfies the cubic equation

$$
y^{3}+A_{2} y^{2}+A_{1} y+B_{0}=0
$$

where $y \equiv g_{4}^{*} \mu_{0} / \mu_{v}, A_{2}$ and $A_{1}$ are given respectively by Eqs. (39) and (40) together with Eq. (43) for the latent heat, $B_{0}$ is given by Eq. (41) with the onset Mach number $M_{k}$ replaced by $M_{4}^{*} \equiv M_{f}\left(x_{4}^{*}\right)>M_{k}$ and $g_{2}^{*}$ is given explicitly by Eq. (42) together with Eq. (43) for the latent heat and with the onset Mach number $M_{k}$ replaced by $M_{2}^{*} \equiv M_{f}\left(x_{2}^{*}\right)>M_{k}$. Figures 5-7 can now be reinterpreted as the predictions $g_{4}^{*}$ as functions of $M_{4}^{*}$ and the predictions $g_{2}^{*}$ as functions of $M_{2}^{*}$. Although $g_{4}^{*}$ and $g_{2}^{*}$ yield respectively better approximations to $g^{*}$ than $g_{3}^{*}$ and $g_{1}^{*}$, for given initial reservoir conditions, nozzle geometry and working fluid, $M_{4}^{*}$ and $M_{2}^{*}$ are not available unless the rate equation is considered. In spite of this fact since both $M_{4}^{*}$ and $M_{2}^{*}$ are greater than $M_{k}$, it seems that better predictions for $g^{*}$ than $g_{2}^{*}$ can be achieved if the onset Mach number $M_{k}$ is increased only slightly. Then the criterion stated above becomes more definite. The exact criterion requires the exact solution of $g^{*}$, which can only be achieved from the exact solution of the rate equation coupled to the equations of flow and state.

\section{Differential theory of nozzle flows with heat addition from phase change and internal sources}

In this section we discuss the differential theory of nozzle flows with heat addition qualitatively by applying the singularity theory of differential equations and show its equivalence with the integro-algebraic theory of the preceding section. By direct differentiation of Eqs. (1)-(4) we arrive at the following system of differential equations:

$$
\begin{aligned}
\frac{1}{p} \frac{d p}{d x}= & -\frac{\gamma M a^{2}}{\Lambda\left(M a, g^{\prime}\right)}\left\{\frac{1}{A} \frac{d A}{d x}+\frac{\frac{\mu_{0}}{\mu_{v}}}{1-\frac{\mu_{0}}{\mu_{v}} g^{\prime}} \frac{d g^{\prime}}{d x}\right. \\
& \left.-\frac{1+\frac{(\gamma-1)}{2} M a^{2}}{1+\frac{q}{c_{p 0} T_{0}}} \frac{d}{d x}\left(\frac{q}{c_{p 0} T_{0}}\right)\right\}
\end{aligned}
$$




$$
\begin{aligned}
& \frac{1}{T} \frac{d T}{d x}=-\frac{\left(1-\frac{\mu_{0}}{\mu_{v}} g^{\prime}\right)(\gamma-1) M a^{2}}{\Lambda\left(M a, g^{\prime}\right)}\left\{\frac{1}{A} \frac{d A}{d x}+\frac{\frac{\mu_{0}}{\mu_{v}}}{1-\frac{\mu_{0}}{\mu_{v}} g^{\prime}} \frac{d g^{\prime}}{d x}\right. \\
& \left.-\frac{\left[\gamma M a^{2}-\left(1-\frac{\mu_{0}}{\mu_{v}} g^{\prime}\right)\right]\left(1+\frac{(\gamma-1)}{2} M a^{2}\right)}{\left(1-\frac{\mu_{0}}{\mu_{v}} g^{\prime}\right)(\gamma-1) M a^{2}\left(1+\frac{q}{c_{p 0} T_{0}}\right)} \frac{d}{d x}\left(\frac{q}{c_{p 0} T_{0}}\right)\right\} \\
& \frac{1}{\varrho} \frac{d \varrho}{d x}=-\frac{M a^{2}\left[1+\frac{\mu_{0}}{\mu_{v}}(\gamma-1) g^{\prime}\right]}{\Lambda\left(M a, g^{\prime}\right)}\left\{\frac{1}{A} \frac{d A}{d x}+\frac{\frac{\mu_{0}}{\mu_{v}}}{M a^{2}\left[1+\frac{\mu_{0}}{\mu_{v}}(\gamma-1) g^{\prime}\right]} \frac{d g^{\prime}}{d x}\right. \\
& \left.-\frac{\left(1-\frac{\mu_{0}}{\mu_{v}} g^{\prime}\right)\left[1+\frac{(\gamma-1)}{2} M a^{2}\right]}{M a^{2}\left[1+\frac{\mu_{0}}{\mu_{v}}(\gamma-1) g^{\prime}\right]\left(1+\frac{q}{c_{p 0} T_{0}}\right)} \frac{d}{d x}\left(\frac{q}{c_{p 0} T_{0}}\right)\right\} \\
& \frac{1}{M a} \frac{d M a}{d x}=\frac{\left(1-\frac{\mu_{0}}{\mu_{v}} g^{\prime}\right)\left[1+\frac{(\gamma-1)}{2} M a^{2}\right]}{\Lambda\left(M a, g^{\prime}\right)}\left\{\frac{1}{A} \frac{d A}{d x}+\frac{\frac{\mu_{0}}{\mu_{v}}}{1-\frac{\mu_{0}}{\mu_{v}} g^{\prime}} \frac{d g^{\prime}}{d x}\right. \\
& \left.-\frac{\left[1-\frac{\mu_{0}}{\mu_{v}} g^{\prime}+\gamma M a^{2}\right]}{2\left(1-\frac{\mu_{0}}{\mu_{v}} g^{\prime}\right)\left(1+\frac{q}{c_{p 0} T_{0}}\right)} \frac{d}{d x}\left(\frac{q}{c_{p 0} T_{0}}\right)\right\}
\end{aligned}
$$

where the Mach number $M a$ and the function $\Lambda\left(M a, g^{\prime}\right)$ are defined by

$$
\begin{aligned}
& M a^{2} \equiv \frac{u^{2}}{\gamma T}, \\
& \Lambda\left(M a, g^{\prime}\right) \equiv M a^{2}\left[1+\frac{\mu_{0}}{\mu_{v}}(\gamma-1) g^{\prime}\right]-\left(1-\frac{\mu_{0}}{\mu_{v}} g^{\prime}\right)
\end{aligned}
$$

and where $q\left(g^{\prime}, x\right)$ is given by Eq. (5). It is important to notice that the isentropic Mach number $M$ introduced in Sections 2.1 and 2.2, differs from the above Mach number $M a$ in that in the definition of the latter the isentropic temperature is replaced by the local temperature. The above 
system of equations reduce to the classical system of differential equations with internal heat addition when $g^{\prime} \equiv 0$ [e.g. see Zierep (1990)] and to the system of differential equations first derived by Barschdorff (1971) for the expansion of wet stream $\left(\omega_{0}=1\right)$ in Laval nozzles when $Q_{\text {in }}^{\prime}(x) \equiv 0$. In the latter case the system of equations is supplemented by the nonequilibrium condensation rate equation. A quantitative solution of the system of Eqs. (45) $-(48)$ requires consideration of the nonequilibrium rate equation if $g^{\prime} \neq 0$ and can only be achieved by numerical analysis. The numerical solutions of the system corresponding to heat addition from internal sources and from nonequilibrium condensation of wet steam are already available in the literature from the work of Jungclaus and van Raay (1967) and Barschdorff (1971). A better understanding of the nature of solution can be achieved qualitatively by applying the singularity theory of differential equations [e.g. see Arnold (1991)]. The application of singularity theory to nozzle flows with heat addition from internal sources was first considered by Möhring (1979) and is discussed in detail by Zierep (1990). We herein generalize this qualitative analysis by applying it to the system of Eqs. (45)-(48). Although a complete qualitative description requires consideration of the nonequilibrium condensation rate equation, we bypass this requirement without loss of generality by assuming that $g^{\prime}(x)$ is an arbitrary, positive, strictly increasing function for $x>0$. Under this hypothesis Eq. (48) plays the key role since Eqs. (45) - (47) can be solved by simple quadrature for given initial reservoir conditions, nozzle geometry and working fluid once the Mach number distribution $M a(x)$ is obtained from Eq. (48).

A qualitative description of Eq. (48) can be performed by identifying its singularities in its phase portrait, the $M a-x$ plane. These singularities are points in the $M a-x$ plane where Eq. (48) is of indeterminate form $0 / 0$. To identify these singularities it is convenient to define

$$
\begin{gathered}
M a^{\star}(x) \equiv \sqrt{\frac{1-\frac{\mu_{0}}{\mu_{v}} g^{\prime}}{1+\frac{\mu_{0}}{\mu_{v}}(\gamma-1) g^{\prime}},} \\
\gamma^{\star}(x) \equiv \frac{\gamma}{1+\frac{\mu_{0}}{\mu_{v}}(\gamma-1) g^{\prime}}, \\
r(x) \equiv \frac{1}{A} \frac{d A}{d x}+\frac{\frac{\mu_{0}}{\mu_{v}}}{1-\frac{\mu_{0}}{\mu_{v}} g^{\prime}} \frac{d g^{\prime}}{d x},
\end{gathered}
$$




$$
s(x) \equiv \frac{1}{2\left(1+\frac{q}{c_{p 0} T_{0}}\right)} \frac{d}{d x}\left(\frac{q}{c_{p 0} T_{0}}\right)
$$

and

$$
h(x) \equiv r(x)-\left[1+\gamma^{\star}(x)\right] s(x)
$$

where $q /\left(c_{p 0} T_{0}\right)=\left[g^{\prime} L^{\prime}+Q_{\text {in }}^{\prime}(x)\right] /\left(c_{p 0}^{\prime} T_{0}^{\prime}\right)$ and $g^{\prime}=g^{\prime}(x)$ is a given strictly increasing function for $x>0$. It is now evident that the singularities of Eq. (48) exist whenever $\Lambda\left(M a, g^{\prime}\right) \rightarrow 0$ and $h(x) \rightarrow 0$ or equivalently $M a \rightarrow M a$ * $(x)$ and $h(x) \rightarrow 0$. It can further be shown that these singularities of Eq. (48) are also precisely the same singularities of Eqs. (45) - (47), thus they are the singularities of the system of equations (45)--(48).

Heat addition from nonequilibrium condensation $\left(g^{\prime} L^{\prime}\right)$ is usually confined to a finite region. If we assume that possible heat addition from other internal sources $\left[Q_{\text {in }}^{\prime}(x)\right]$ is also confined to a finite region and that the total heat addition over this finite region is without oscillations, then typical behavior of the functions $r(x)$ and $\left[1+\gamma^{\star}(x)\right] s(x)$ is as shown in Figs. 9(a) and (b). Figure 9(a) shows the case of single singular point, the classical saddle point at a throat (subcritical flow). Figure 9(b) shows three zeros $0=x_{1}<x_{2}<x_{3}$ of $h$ corresponding to three singularities of Eq. (48) where the Mach number $M a$ takes the values $M a^{\star}\left(x_{n}\right) n=1,2,3$. To investigate the nature of these singularities we proceed with further analysis. By applying L'Hôpital's Rule to Eq. (48) we arrive at

$$
2 \psi^{2}+\left[\gamma_{n}^{\star}\left(\frac{1}{A} \frac{d A}{d x}\right)_{n}+\frac{2 \gamma_{n}^{\star}\left(\frac{\mu_{0}}{\mu_{v}}\right)}{1-\frac{\mu_{0}}{\mu_{v}} g_{n}^{\prime}}\left(\frac{d g^{\prime}}{d x}\right)_{n}\right] \psi-\frac{1}{2} G=0
$$

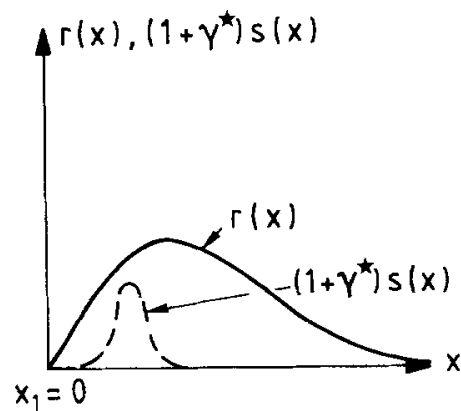

(a)

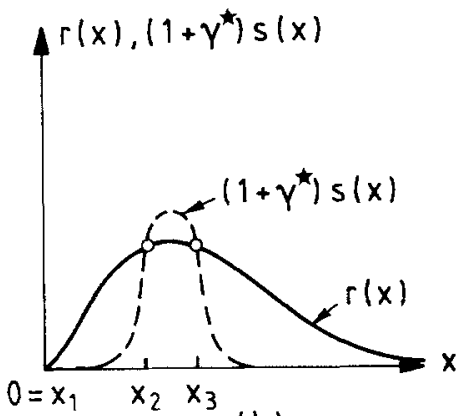

(b)

Figure 9

Typical behavior of the functions $r(x)$ and $\left[1+\gamma^{\star}(x)\right] s(x)$ possessing (a) a single singularity (b) three singularities of Eq. (48). 
where

$$
\begin{aligned}
G \equiv\left(\gamma_{n}^{\star}+1\right)\left(\frac{d h}{d x}\right)_{n}-\frac{\gamma_{n}^{\star}\left(\frac{\mu_{0}}{\mu_{v}}\right)\left(\frac{d g^{\prime}}{d x}\right)_{n}\left(\frac{1}{A} \frac{d A}{d x}\right)_{n}}{1-\frac{\mu_{0}}{\mu_{v}} g_{n}^{\prime}} \\
-\gamma_{n}^{\star}\left[\frac{\frac{\mu_{0}}{\mu_{v}}\left(\frac{d g^{\prime}}{d x}\right)_{n}}{1-\frac{\mu_{0}}{\mu_{v}} g_{n}^{\prime}}\right]^{2}, \\
\psi \equiv \lim _{(h, M a) \rightarrow\left(0, M a^{*}(x)\right)} \frac{1}{M a} \frac{d M a}{d x},
\end{aligned}
$$

$\gamma_{n}^{\star} \equiv \gamma^{\star}\left(x_{n}\right)$ and where by subscript $n$ we mean 'evaluated at $x=x_{n}$ $n=1,2,3$ '. The solution of Eq. (56) yields

$$
\psi_{1,2}=\frac{-\left[\gamma_{n}^{\star}\left(\frac{1}{A} \frac{d A}{d x}\right)_{n}+\frac{2 \gamma_{n}^{\star}\left(\mu_{0} / \mu_{v}\right)\left(d g^{\prime} / d x\right)_{n}}{1-\left(\mu_{0} / \mu_{v}\right) g_{n}^{\prime}}\right] \pm \sqrt{D}}{4}
$$

where

$$
D \equiv\left[\gamma_{n}^{\star}\left(\frac{1}{A} \frac{d A}{d x}\right)_{n}+\frac{2 \gamma_{n}^{\star}\left(\frac{\mu_{0}}{\mu_{v}}\right)\left(\frac{d g^{\prime}}{d x}\right)_{n}}{1-\frac{\mu_{0}}{\mu_{v}} g_{n}^{\prime}}\right]^{2}+4 G
$$

A discussion of Eq. (59) reveals that a singularity $x_{n}$ of Eq. (48) is

(i) a saddle point with or without heat addition if $G \geq 0$ and $D>0$,

(ii) a nodal point with heat addition if $G<0$ and $D \geq 0$,

(iii) a turning point without heat addition if $G=D=0$,

(iv) a spiral point with heat addition if $G<0$ and $D<0$ and

(v) a vortex point without heat addition if $G<0$ and $D<0$.

The local behavior of the singularities of Eq. (48) are exhibited in Figs. 10 (a) -(f) in the $M a-x$ plane where the origin is chosen at the singular point $\left(x_{n}, M a_{n}^{\star}\right)$ with $M a_{n}^{\star} \equiv M a^{\star}\left(x_{n}\right)$.

We can now discuss the possible global solutions of Eq. (48) from the local behavior of Eq. (59) by noting that

(i) $x_{1}=0$ is a saddle point without heat addition (classical throat with $G>0, D>0$ ),

(ii) $x_{3}$ is a saddle point with or without heat addition $(G>0, D>0)$ and

(iii) $x_{2}$ is either a spiral point $(G<0, D<0)$ or a nodal point $(G<0, D \geq 0)$ with heat addition. 


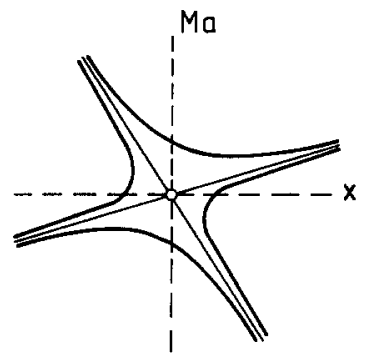

(a)

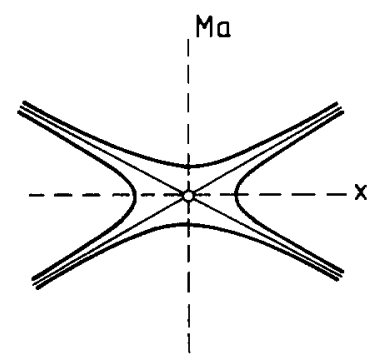

(d)

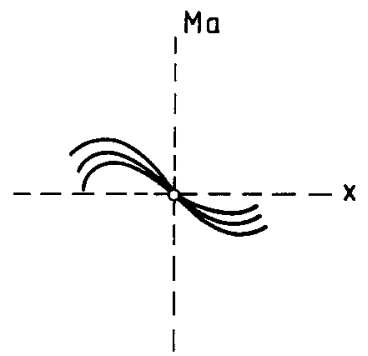

(b)

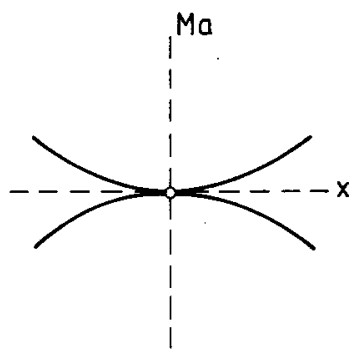

(e)

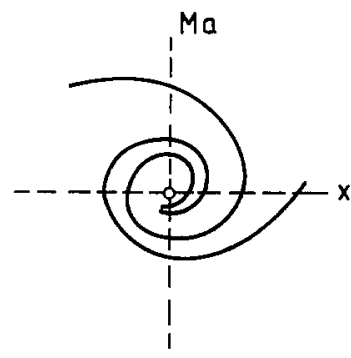

(c)

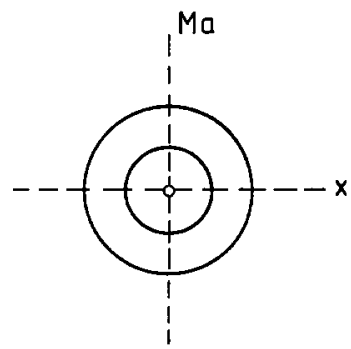

(f)

Figure 10

Classification of singularities of Eq. (48). (a) a saddle point with heat addition, (b) a nodal point with heat addition, (c) a spiral point with heat addition, (d) a saddle point without heat addition, (e) a turning point without heat addition, (f) a vortex point without heat addition.

Figures 11 and 12 show possible global solutions of Eq. (48) where $x_{2}$ is a spiral point and a nodal point respectively and $x_{3}$ is a saddle point with or without heat addition. In both cases the flow is eventually accelerated to supersonic speeds. In addition Fig. 11 predicts a unique position for the shock wave to be fitted when $x_{2}$ is a spiral point.

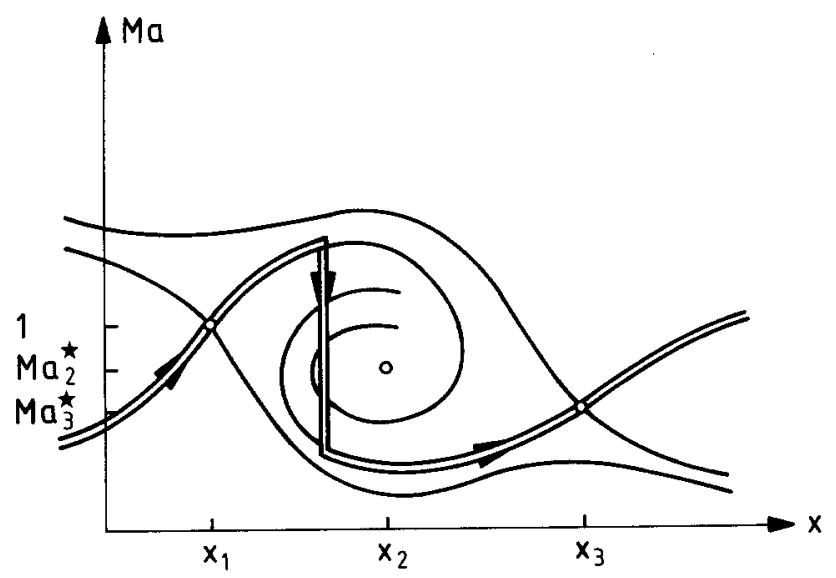

Figure 11

Global solution of Eq. (48) when $x_{2}$ is a spiral point and $x_{3}$ is a saddle point with or without heat addition. 


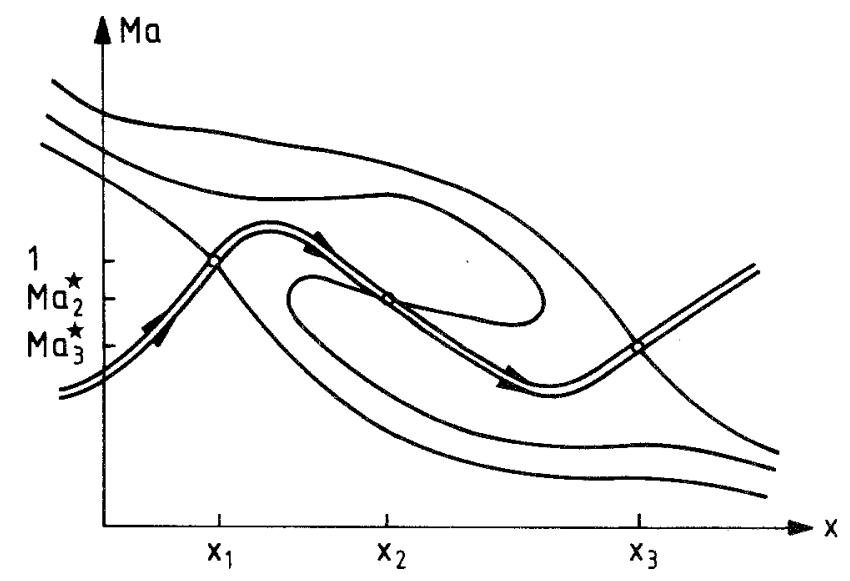

Figure 12

Global solution of Eq. (48) when $x_{2}$ is a nodal point and $x_{3}$ is a saddle point with or without heat addition.

Having discussed the global solution of the system of differential equations (45) - (48) qualitatively by its singularities, we now investigate the equivalent conditions at the singularities in the integro-algebraic theory. It follows at once from Eqs. (7) and (8) that $M a \rightarrow M a^{\star}(x)$ as $\Delta \rightarrow 0$ which shows that the condition $M a \rightarrow M a^{\star}(x)$ in differential theory corresponds to the critical flow condition $\Delta \rightarrow 0$ or $q /\left(c_{p 0} T_{0}\right) \rightarrow q^{*} /\left(c_{p 0} T_{0}\right)$ in the integroalgebraic formulation. Furthermore it can be shown after cumbersome manipulations that the condition $h \rightarrow 0$ in differential theory is equivalent to the condition $d / d x\left(q / c_{p 0} T_{0}\right) \rightarrow d / d x\left(q^{*} / c_{p 0} T_{0}\right)$ in integro-algebraic theory. Therefore the conditions

$$
M a=M a^{\star}(x) \text { and } h=0
$$

that identify the singularities in differential theory are equivalent to the conditions

$$
\frac{q}{c_{p 0} T_{0}}=\frac{q^{*}}{c_{p 0} T_{0}} \text { and } \frac{d}{d x}\left(\frac{q}{c_{p 0} T_{0}}\right)=\frac{d}{d x}\left(\frac{q^{*}}{c_{p 0} T_{0}}\right)
$$

in integro-algebraic theory. This proves that the flow necessarily reaches critical flow at the singularities of the differential system.

\section{Thermal choking}

The phenomenon of thermal choking in nozzle flows is usually attributed to heat addition from phase change or internal sources exceeding a certain critical amount, which is now exactly defined by Eqs. (20) and (29) respectively for diabatic flows and for flows with nonequilibrium condensa- 
tion. It is commonly believed that thermal choking occurs when the compressive effect produced by heat addition overweighs the influence of increasing cross-section moving the flow Mach number ${ }^{2}$ toward unity. Thus a commonly accepted criterion for thermal choking [e.g. Pouring (1965)] is tied up with the critical flow condition which presumably occurs when the flow Mach number reaches unity. In such a case the flow is visualized by the occurrence of normal shock waves.

Now that we have defined the exact critical flow conditions for diabatic nozzle flows and for nozzle flows with nonequilibrium condensation, we investigate the nature of thermal choking by identifying the necessary and sufficient conditions for its occurrence. For a precise definition of thermal choking we rely upon experimental observations that it is visualized by the occurrence of normal shock waves. We call a flow thermally choked if no continuous solution of Eqs. (1)-(4) exists for given initial reservoir conditions, nozzle geometry and working fluid by assuming that $Q_{\text {in }}^{\prime}(x)$ and $g^{\prime}(x)$ are arbitrary strictly increasing functions for $x>0$ (supersonic heat addition). It then follows immediately from the integro-algebraic theory that $\Delta=0$ (or $Q /\left(c_{p 0} T_{0}\right)=Q^{*} /\left(c_{p 0} T_{0}\right)$ for diabatic flows and $q /\left(c_{p 0} T_{0}\right)=q^{*} /\left(c_{p 0} T_{0}\right)$ for flows with nonequilibrium condensation) or equivalently from the differential theory that $M a=M a^{\star}(x)$ is a necessary condition for thermal choking. Ruling out the possibilities for the occurrence of a continuous global solution of the preceding section we arrive at the conclusion that the flow is thermally choked if and only if the system of flow equations exhibits a spiral singularity. Thus the necessary and sufficient conditions for a flow to be thermally choked can be identified as
(a) $\frac{q}{c_{p 0} T_{0}}=\frac{q^{*}}{c_{p 0} T_{0}}$ or equivalently $M a=M a^{\star}(x)$,
(b) $\frac{d}{d x}\left(\frac{q}{c_{p 0} T_{0}}\right)=\frac{d}{d x}\left(\frac{q^{*}}{c_{p 0} T_{0}}\right)$ or equivalently $h=0$ and
(c) $G<0, D<0$

at some point $\left(\hat{x}, M a^{\star}(\hat{x})\right)$ in $M a-x$ plane. This suggests that a thermally choked flow is supercritical and vice versa. It can further be demonstrated that a sufficient condition for thermal choking is

$$
\frac{d M a}{d x} \rightarrow-\infty \quad \text { as } \quad M a \rightarrow M a^{\star}(x)
$$

for some $x=x^{\star}$.

2 For nozzle flows with nonequilibrium condensation this is the local frozen Mach number. 
Having exhibited the necessary and sufficient conditions for thermal choking, we now show that the commonly accepted criterion of thermal choking which states that the flow Mach number reaches unity (which may not be true in general) is only a necessary condition. To demonstrate this fact we first define the local frozen speed of sound by

$$
a_{f}^{2} \equiv-\frac{h_{\varrho}}{h_{p}-\frac{1}{\varrho}}
$$

following Vincenti and Kruger (1965) where

$$
h=c_{p 0} T-L(T) \frac{\mu_{0}}{\mu_{v}} g^{\prime}-Q(x)
$$

with

$$
L(T) \equiv \frac{L^{\prime}(T) \mu_{v}}{\mathfrak{R} T_{s}^{\prime}} \quad \text { and } \quad Q(x) \equiv \frac{Q_{i n}^{\prime}(x) \mu_{0}}{\mathfrak{R} T_{s}^{\prime}} .
$$

If we denote the local frozen Mach number (the flow Mach number) by

$$
M_{g}^{2} \equiv \frac{u^{2}}{a_{f}^{2}},
$$

we can easily show that it is related to the Mach number $M a$ by

$$
M_{g}^{2}=M a^{2} \frac{\gamma\left[1+\frac{\mu_{0}}{\mu_{v}} g^{\prime}(\gamma-1)\left(1-L_{1}\right)\right]}{\left[\gamma-L_{1} g^{\prime} \frac{\mu_{0}}{\mu_{v}}(\gamma-1)\right]\left[1-\frac{\mu_{0}}{\mu_{v}} g^{\prime}\right]}
$$

where $L_{1}(T) \equiv d L / d T$. Now a necessary condition for thermal choking ( $M a=M a^{\star}(x)$ at some $x=x^{\star}$ or $g^{\prime}=g^{\star}$ where $L_{1}$ takes the value $\left.L_{1}^{\star}\right)$ in terms of the flow Mach number becomes

$M_{g}=M_{g}^{\star} \equiv \sqrt{\frac{1-\gamma^{\star} \zeta^{\star}}{1-\zeta^{\star}}}$

where

$$
\zeta^{\star} \equiv \frac{\mu_{0}}{\mu_{v}} \frac{(\gamma-1)}{\gamma} g^{\star} L_{1}^{\star}
$$

and

$$
\gamma^{\star} \equiv \frac{\gamma}{1+\frac{\mu_{0}}{\mu_{v}} g^{\star}(\gamma-1)} .
$$


It is important to note that for the widely adopted two-phase homogeneous dispersed flow model of liquid droplets and gas mixture (with the classical assumptions that no slip between the dispersed droplets and the mixture of gases is taken into account, the enthalpy difference of the liquid and gaseous phases is approximated by the latent heat of condensation, etc.), also assumed herein, the commonly accepted view that the flow Mach number $M_{g}$ reaches unity at thermal choking is only true if $\zeta^{\star} \rightarrow 0$ (i.e. $L_{1} \rightarrow 0$, the case of constant latent heat). It is now obvious from Eq. (64) that $M_{g}^{\star}<1$ whenever $0<\zeta^{\star}<1 / \gamma^{\star}$ and $M_{g}^{\star}>1$ whenever $\zeta^{\star}<0$. However, since the latent heat is weakly dependent on temperature for most working fluids in the operational range of the nozzle, it follows that $\left|\zeta^{\star}\right| \ll 1$ which shows that the classical view that $M_{g}^{\star} \rightarrow 1$ for a thermally choked flow is almost true and is realized within the accuracy of experimental visualization.

\section{Discussion}

In this investigation the integro-algebraic and differential formulations of the flow and state equations for nozzle flows with heat addition from phase change and other internal sources are employed to analyze the phenomenon of thermal choking. The exact expressions that define the critical amount of heat necessarily attained at thermal choking are respectively given by Eqs. (20) and (29) for diabatic nozzle flows and nozzle flows with nonequilibrium condensation. By application of the singularity theory to the system of differential equations for nozzle flows with or without heat addition, the singularities of the system are classified (Fig. 10) and the topological phase portraits of possible flow patterns with heat addition reaching the critical amount are exhibited (Figs. 11 and 12). A thermally choked or supercritical flow is identified as the flow pattern which is visualized by the occurrence of normal shock waves and the necessary and sufficient conditions at thermal choking are displayed in Section 4 as the conditions for the existence of a spiral singularity. It is then shown that the flow Mach number at thermal choking is not necessarily unity in condensing nozzle flows due to the variation from the temperature dependence of the latent heat.

In predicting thermal choking in nozzle flows with nonequilibrium condensation, approximate models that evaluate the critical amount of heat are constructed as presented in Section 2.3. Consequently a cubic equation [Eq. (38)] which relates the estimate $g_{3}^{*}$ of the limiting condensate mass fraction to the onset Mach number $M_{k}$ (presumably to be evaluated by the Zierep and Lin [1967] similarity law using empirical exponents for a given working fluid) is derived and a criterion for the existence of supercritical condensing flows based on this estimate is established. This criterion for 
thermal choking is tested against data of moist air expansions from nozzle experiments conducted recently by Schnerr (1986) and good agreement is achieved. For other working fluids (e.g. pure steam) the applicability of this criterion can easily be determined when satisfactory data from experiments with these fluids are available.

In all of the above treatment both the condensible vapor and the carrier gas constituting the mixture are assumed to be perfect gases. It would be interesting to find out whether the above analytical treatment of thermal choking can be extended to account for real gas effects. Even if a simple equation such as the van der Waals equation of state is adopted for the condensible vapor phase alone, arriving at a solution of the flow and state equations in functional form, such as Eqs. (7)-(11) for a mixture of perfect gases, from which the critical amount of heat can be identified seems not possible.

\section{Acknowledgements}

The authors would like to thank Prof. P. P. Wegener of Yale University for a fruitful brief discussion. One of the authors (C.F.D.) acknowledges the support in the form of a Research Fellowship by the Alexander von Humboldt Foundation during his stay in Karlsruhe.

\section{References}

Arnold, V. I., Gewöhnliche Differentialgleichungen (translated from Russian). Deutscher Verlag der Wissenschaften, Berlin 1991.

Barschdorff, D., Kurzzeitfeuchtemessung und ihre Anwendung bei Kondensationserscheinungen in Lavaldüsen. Strömungsmechanik and Strömungsmaschinen. Mitt. d. Inst. f. Strömungslehre und Strömungsmaschinen, Heft 6, Universität Karlsruhe 1967.

Barschdorff, D., Verlauf der Zustandsgrößen und gasdynamische Zusammenhänge bei der spontanen Kondensation reinen Wasserdampfes in Lavaldüsen. Forsch. Ing.-Wes. 37(5), 146-156 (1971).

Blythe, P. A. and Shih, C. J., Condensation shocks in nozzle flows. J. Fluid Mech. 76, 593-621 (1976).

Clarke, J. H. and Delale, C. F., Nozzle flows with nonequilibrium condensation. Phys. Fluids 29(5), $1398-1413$ (1986).

Hill, P. G., Condensation of water vapour during supersonic expansion in nozzles. J. Fluid Mech. 35 , $593-620(1966)$.

Jungclaus, G. and van Raay, O., Berechnung der Strömung in Lavaldüsen mit beliebig verteilter Wärmezufuhr. Ingenieur-Archiv 36, 226-236 (1967).

Möhring, W., On flows with heat addition in Laval nozzles. In Recent Developments in Theoretical and Experimental Fluid Mechanics. Springer-Verlag, Berlin/Heidelberg/New York 1979, pp. 179-185.

Pouring, A. A., Thermal choking and condensation in nozzles. Phys. Fluids 8(10), 1802-1810 (1965).

Schnerr, G. H., Homogene Kondensation in stationären transsonischen Strömungen durch Lavaldüsen und um Profile. Habilitationsschrift Fakultät für Maschinenbau, Universität (TH) Karlsruhe 1986.

Schnerr, G. H., 22-D transonic flow with energy supply by homogeneous condensation. Experiments in Fluids 7, 145-156 (1989).

Schnerr, G. H. and Dohrmann, U., Theoretical and Experimental Imestigation of 2-D Diabatic Transonic and Supersonic Flow Fields. Proc. of IUTAM Symposium Transsonicum III (eds. Zierep, J. and Oertel, H.), Springer-Verlag, Berlin/Heidelberg/New York 1989, pp. 125-135.

Shapiro, A. H., The Dynamics and Thermodynamics of Compressible Fluid Flow. Vols. 1 and 2 , Ronald Press Co., New York 1953. 
Thompson, P. A., Compressible Fluid Dynamics. McGraw-Hill, New York 1972.

Vincenti, W. G. and Kruger, C. H., Introduction to Physical Gas Dynamics. Wiley \& Sons, New York/London/Sydney 1965.

Wegener, P. P., Gas dynamics of expansion flows with condensation and homogeneous nucleation of water vapor. In Nonequilibrium Flows - Part 1 (ed. Wegener, P. P.), Marcel Dekker, New York/London 1969, pp. 163-243.

Wegener, P. P. and Mack, L. M., Condensation in supersonic and hypersonic wind tunnels. Advances in Appl. Mech. 5, 307-447 (1958).

Wegener, P. P. and Pouring, A. A., Experiments on condensation of water vapor by homogeneous nucleation in nozzles. Phys. Fluids 7, 352-361 (1958).

Zierep, J., Ähnlichkeitsgesetze für Profilströmungen mit Wärmezufuhr. Acta Mech. 1, 60-70 (1965).

Zierep, J., Strömungen mit Energiezufuhr. G. Braun, Karlsruhe 1990.

Zierep, J. and Lin, S., Bestimmung des Kondensationsbeginns bei Entspannung feuchter Luft in Überschalldüsen. Forsch. Ing.-Wes. 33, 169-172 (1967).

\begin{abstract}
The mathematical theory of sub- and supercritical nozzle flows is presented by a unified description of integro-algebraic and differential formulations of the flow equations. The critical amount of heat necessary for a thermally choked flow is defined and models which approximate this critical amount of heat are constructed for nozzle flows with both given internal heat source distributions and nonequilibrium condensation. In particular a cubic equation for an estimate of the limiting condensate mass fraction for shock free condensing flows is derived and a criterion for the existence of supercritical condensing flows based on this estimate is established. The necessary and sufficient conditions for thermal choking are then stated. It is shown that the commonly accepted view, which asserts that the flow Mach number reaches unity at thermal choking (known to be not always true in condensing flows), only exhibits a necessary condition for a thermally choked flow.
\end{abstract}

(Received: October 20, 1992) 